\title{
Molecular regimes in ultracold Fermi gases
}

\author{
D.S. Petrov $^{1,2}$, C. Salomon ${ }^{3}$, and G.V. Shlyapnikov ${ }^{1,4}$ \\ ${ }^{1}$ Laboratoire de Physique Théorique et Modèles Statistiques, Université Paris- \\ Sud, 91405, Orsay Cedex, France \\ ${ }^{2}$ Russian Research Center, Kurchatov Institute, Kurchatov Square, 123182 Moscow, \\ Russia \\ ${ }^{3}$ Laboratoire Kastler Brossel, Ecole Normale Supérieure, 24 rue Lhomond, 75231, \\ Paris, France \\ ${ }^{4}$ Van der Waals-Zeeman Institute, University of Amsterdam, Valckenierstraat \\ 65/67, 1018 XE Amsterdam, The Netherlands
}

\begin{abstract}
The use of Feshbach resonances for tuning the interparticle interaction in ultracold Fermi gases has led to remarkable developments, in particular to the creation and Bose-Einstein condensation of weakly bound diatomic molecules of fermionic atoms. These are the largest diatomic molecules obtained so far, with a size of the order of thousands of angstroms. They represent novel composite bosons, which exhibit features of Fermi statistics at short intermolecular distances. Being highly excited, these molecules are remarkably stable with respect to collisional relaxation, which is a consequence of the Pauli exclusion principle for identical fermionic atoms. The purpose of this review is to introduce theoretical approaches and describe the physics of molecular regimes in two-component Fermi gases and Fermi-Fermi mixtures, focusing attention on quantum statistical effects.
\end{abstract}

\section{Contents}

1 Introduction 2

1.1 State of the art ....................... 2

1.2 Feshbach resonances and diatomic molecules . . . . . . . . . 4

2 Homonuclear diatomic molecules in Fermi gases 7

2.1 Weakly interacting gas of bosonic molecules. Molecule-molecule elastic interaction . . . . . . . . . . . . . 7

2.2 Suppression of collisional relaxation . . . . . . . . . . . . 12

2.3 Collisional stability and molecular BEC . . . . . . . . . . 15

3 Heteronuclear molecules in Fermi-Fermi mixtures 17

3.1 Effect of mass ratio on elastic intermolecular interaction . . . . 17 
3.2 Collisional relaxation for moderate mass ratios . . . . . . . . 20

3.3 Born-Oppenheimer picture of collisional relaxation . . . . . . . . 21

3.4 Molecules of heavy and light fermionic atoms . . . . . . . . . . 23

3.5 Trimer states . . . . . . . . . . . . . . . . . 26 26

3.6 Collisional relaxation of molecules of heavy and light fermions and formation of trimers . . . . . . . . . 28

4 Crystalline molecular phase

4.1 Born-Oppenheimer potential in a many-body system of molecules of heavy and light fermions . . . . . . . . . . . . 34

4.2 Gas-crystal quantum transition . . . . . . . . . . . . . . . 37

4.3 Molecular superlattice in an optical lattice . . . . . . . . . 37

5 Concluding remarks and prospects

\section{Introduction}

\subsection{State of the art}

The field of quantum gases is rapidly expanding in the direction of ultracold clouds of fermionic atoms, with the goal of revealing novel macroscopic quantum states and achieving various regimes of superfluidity. The initial idea was to achieve the Bardeen-Cooper-Schrieffer (BCS) superfluid phase transition in a two-component Fermi gas, which requires attractive interactions between the atoms of different components. Then, in the simplest version of this transition, at sufficiently low temperatures fermions belonging to different components and with opposite momenta on the Fermi surface form correlated (Cooper) pairs in the momentum space. This leads to the appearance of a gap in the singleparticle excitation spectrum and to the phenomenon of superfluidity (see, for example, [1]). In a dilute ultracold two-component Fermi gas, most efficient is the formation of Cooper pairs due to the attractive intercomponent interaction in the s-wave channel (negative $s$-wave scattering length $a$ ). However, for typical values of $a$, the superfluid transition temperature is extremely low. For this reason, the efforts of many experimental groups have been focused on modifying the intercomponent interaction using Feshbach resonances. The scattering length $a$ near a Feshbach resonance can be tuned from $-\infty$ to $+\infty$. This has led to exciting developments (see 2 for review), such as the direct observation of superfluid behavior in the strongly interacting regime $\left(n|a|^{3} \gtrsim 1\right.$, where $n$ is the gas density) through vortex formation [3, and the study of the influence of imbalance between the two components of the Fermi gas on superfluidity [4, 5, 6, 7, 8.

We focus here on the remarkable physics of weakly bound diatomic molecules of fermionic atoms. This initially unexpected physics connects molecular and condensed matter physics. The weakly bound molecules are formed on the positive side of the resonance $(a>0), 9,10,11,12$ and they are the largest diatomic 
molecules obtained so far. Their size is of the order of $a$ and it reaches thousands of angstroms in current experiments. Accordingly, their binding energy is exceedingly small ( $10 \mu \mathrm{K}$ or less). Being composite bosons, these molecules obey Bose statistics, and they have been Bose-condensed in experiments with ${ }^{40} \mathrm{~K}_{2}$ 13, 14, at JILA and with ${ }^{6} \mathrm{Li}_{2}$ at Innsbruck [15, 16, MIT [17, 18, ENS [19, Rice [20, and Duke 21]. Nevertheless, some of the interaction properties of these molecules reflect Fermi statistics of the individual atoms forming the molecule. In particular, these molecules are found to be remarkably stable with respect to collisional decay. Being in the highest rovibrational state, they do not undergo collisional relaxation to deeply bound states on a time scale exceeding seconds at densities of about $10^{13} \mathrm{~cm}^{-3}$. This is more than four orders of magnitude longer than the life time of similar molecules consisting of bosonic atoms. The key idea of our discussion of homonuclear diatomic molecules formed in a two-component Fermi gas by atoms in different internal (hyperfine) states is to show how one obtains an exact universal result for the elastic interaction between such weakly bound molecules and how the Fermi statistics for the atoms provides a strong suppression of their collisional relaxation into deep bound states. It is emphasized that the repulsive character of the elastic intermolecular interaction and remarkable collisional stability of the molecules are the main factors allowing for their Bose-Einstein condensation and for prospects related to interesting manipulations with these molecular condensates.

Currently, a new generation of experiments is being developed for studying degenerate mixtures of different fermionic atoms [22, 23, with the idea of revealing the influence of the mass difference on superfluid properties and finding novel types of superfluid pairing. On the positive side of the resonance one expects the formation of heteronuclear weakly bound molecules, which attracts a great deal of interest, in particular for creating dipolar gases. We present an analysis of how the mass ratio for constituent atoms influences the elastic interaction between the molecules and their collisional stability. The discussion is focused on molecules of heavy and light fermions, where one expects the formation of trimer bound states and the manifestation of the Efimov effect. We then show that a many-body system of such molecules can exhibit a gas-crystal quantum transition. Remarkably, the atomic system itself remains dilute, and the crystalline ordering is due to a relatively long-range interaction between the molecules originating from exchange of light fermions. Realization of the crystalline phase requires a very large mass ratio for the atoms forming a molecule in order to suppress the molecular kinetic energy. This can be achieved in an optical lattice for heavy atoms, where the crystalline phase of a dilute molecular system emerges as a superlattice, and we discuss the related physics.

The chapter is concluded by an overview of prospects for manipulations with the weakly bound molecules of fermionic atoms. The leading ideas include the achievement of ultra-low temperatures and BCS transition for atomic fermions, creation of dipolar quantum gases, as well as observation of peculiar trimer bound states in an optical lattice. 


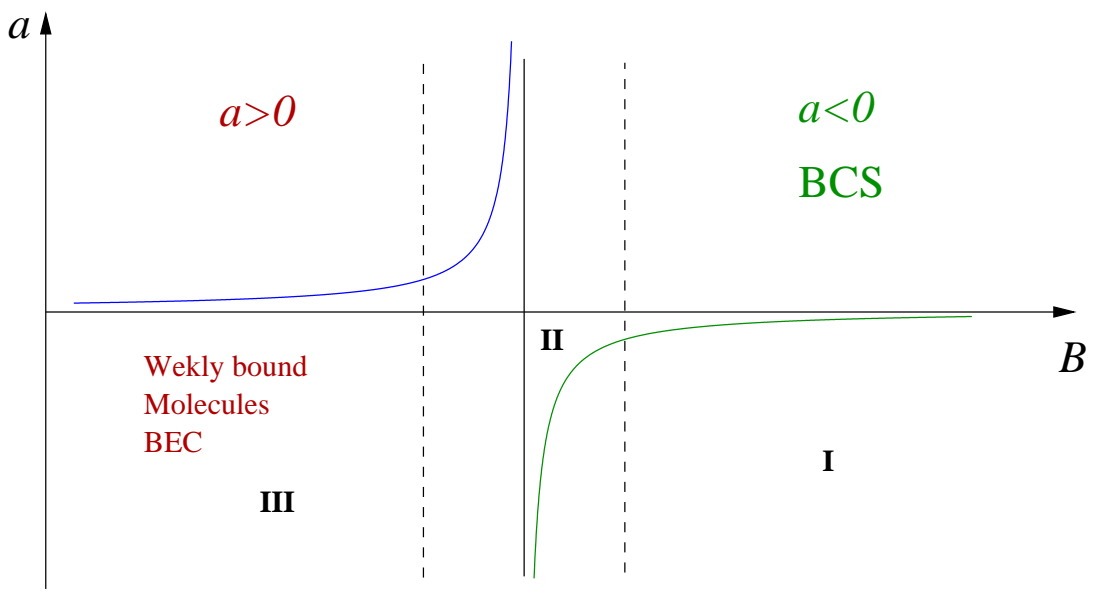

Figure 1: The dependence of the scattering length on the magnetic field near a Feshbach resonance. The symbols I, II, and III label the regime of a weakly interacting degenerate atomic Fermi gas, strongly interacting regime of BCS$\mathrm{BEC}$ crossover, and the regime of weakly bound molecules. At sufficiently low temperatures region I corresponds to the BCS superfluid pairing, and region III to Bose-Einstein condensation of molecules.

\subsection{Feshbach resonances and diatomic molecules}

At ultralow temperatures, when the de Broglie wavelength of atoms greatly exceeds the characteristic radius of interatomic interaction forces, atomic collisions and interactions are generally determined by the $s$-wave scattering. Therefore, in two-component Fermi gases one may consider only the interaction between atoms of different components, which can be tuned by using Feshbach resonances.

The description of a many-body system near a Feshbach resonance requires a detailed knowledge of the 2-body problem. In the vicinity of the resonance, the energy of a colliding pair of atoms in the open channel is close to the energy of a molecular state in another hyperfine domain (closed channel). The coupling between these channels leads to a resonant dependence of the scattering amplitude on the detuning $\delta$ of the closed channel state from the threshold of the open channel, which can be controlled by an external magnetic (or laser) field. Thus, the scattering length becomes field dependent (see Fig. 1).

The Feshbach effect is a two-channel problem which can be described in terms of the Breit-Wigner scattering [24, 25, and various aspects of such problems have been discussed by Feshbach [26] and Fano [27. In cold atom physics the idea of Feshbach resonances was introduced in Ref. [28, and optically induced resonances have been analyzed in Refs. [29, 30, 31, 32.

At resonance the scattering length changes from $+\infty$ to $-\infty$, and in the vicinity of the resonance one has the inequality $n|a|^{3} \gtrsim 1$ where $n$ is the gas 
density. The gas is ten said to be in the strongly interacting regime. It is still dilute in the sense that the mean interparticle separation greatly exceeds the characteristic radius of the interparticle interaction $R_{e}$. However, the amplitude of binary interactions (scattering length) is larger than the mean separation between particles, and in the quantum degenerate regime the conventional mean field approach is no longer valid.

For large detuning from resonance the gas is in the weakly interacting regime, i.e. the inequality $n|a|^{3} \ll 1$ is satisfied. On the negative side of the resonance $(a<0)$, at sufficiently low temperatures of the two-species Fermi gas one expects the BCS pairing between distinguishable fermions, well described in literature 11. On the positive side $(a>0)$ two fermions belonging to different components form diatomic molecules. For $a \gg R_{e}$ these molecules are weakly bound and their size is of the order of $a$.

The crossover from the BCS to BEC behavior has recently attracted now a great deal of interest, in particular with respect to the nature of superfluid pairing, transition temperature, and elementary excitations. This type of crossover has been earlier discussed in the literature in the context of superconductivity [33, 34, 35, 36] and in relation to superfluidity in two-dimensional films of ${ }^{3} \mathrm{He}$ [37, 38. The idea of resonant coupling through a Feshbach resonance for achieving a superfluid phase transition in ultracold two-component Fermi gases has been proposed in Refs. 39, 40, and for the two-dimensional case it has been discussed in Ref. 41.

The two-body physics of the Feshbach resonance is the most transparent if the (small) background scattering length is neglected. Then for low collision energies $\varepsilon$ the scattering amplitude is given by [25] :

$$
F(\varepsilon)=-\frac{\hbar \gamma / \sqrt{2 \mu}}{\varepsilon+\delta+i \gamma \sqrt{\varepsilon}},
$$

where the quantity $\hbar \gamma / \sqrt{2 \mu} \equiv W$ characterizes the coupling between the open and closed channels and $\mu$ is the reduced mass of the two atoms. The scattering length is $a=-F(0)$. In Eq. (1) the detuning $\delta$ is positive if the bound molecular state is below the continuum of the colliding atoms. Then for $\delta>0$ the scattering length is positive, and for $\delta<0$ it is negative. Introducing a characteristic length

$$
R^{*}=\hbar^{2} / 2 \mu W
$$

and expressing the scattering amplitude through the relative momentum of particles $k=\sqrt{2 \mu \varepsilon} / \hbar$, we can rewrite Eq. (1) in the form:

$$
F(k)=-\frac{1}{a^{-1}+R^{*} k^{2}+i k} .
$$

The validity of Eq. (3) does not require the condition $k R^{*} \ll 1$. At the same time, this equation formally coincides with the amplitude of scattering of slow particles by a potential with the same scattering length $a$ and an effective range $R=-2 R^{*}$, obtained under the condition $k|R| \ll 1$. 
The length $R^{*}$ is an intrinsic parameter of a Feshbach resonance. It characterizes the width of the resonance. From Eqs. (1) and (2) we see that small $W$ and, consequently, large $R^{*}$ correspond to narrow resonances, whereas large $W$ and small $R^{*}$ lead to wide resonances. The term "wide" is generally used when the length $R^{*}$ drops out of the problem, which according to Eq. (3) requires the condition $k R^{*} \ll 1$. In a quantum degenerate atomic Fermi gas the characteristic momentum of particles is the Fermi momentum $k_{F}=\left(3 \pi^{2} n\right)^{1 / 3}$. Thus, in the strongly interacting regime and on the negative side of the resonance $(a<0)$, for a given $R^{*}$ the condition of the wide resonance depends on the gas density $n$ and takes the form $k_{F} R^{*} \ll 1$ [42, 43, 44, 45, 46].

For $a>0$ one has weakly bound molecular states (it is certainly assumed that the characteristic radius of interaction $R_{e} \ll a$ ), and for such molecular systems the criterion of the wide resonance is different [47, 49]. The binding energy of the weakly bound molecule state is determined by the pole of the scattering amplitude (3). One then finds [47, 49] that this state exists only for $a>0$, and under the condition

$$
R^{*} \ll a
$$

the binding energy is given by

$$
\varepsilon_{0}=\hbar^{2} / 2 \mu a^{2} .
$$

The wavefunction of such weakly bound molecular state has only a small admixture of the closed channel, and the size of the molecule is $\sim a$. The characteristic momenta of the atoms in the molecule are of the order of $a^{-1}$ and in this respect the inequality (4) represents the criterion of a wide resonance for the molecular system.

Under these conditions atom-molecule and molecule-molecule interactions are determined by a single parameter - the atom-atom scattering length $a$. In this sense, the problem becomes universal. It is equivalent to the interaction problem for the two-body potential which is characterized by a large positive scattering length $a$ and has a potential well with a weakly bound molecular state. The picture remains the same when the background scattering length can not be neglected, although the condition of a wide resonance can be somewhat modified [50].

Most ongoing experiments with Fermi gases of atoms in two different internal (hyperfine) states use wide Feshbach resonances [51. For example, weakly bound molecules ${ }^{6} \mathrm{Li}_{2}$ and ${ }^{40} \mathrm{~K}_{2}$ have been produced in experiments [10, 11, 12, 13, 14, 17, 18, 15, 16, 19, 20 by using Feshbach resonances with a length $R^{*} \lesssim 20 \AA$, and for the achieved values of the scattering length $a$ (from 500 to $2000 \AA$ ) the ratio $R^{*} / a$ was smaller than 0.1 . In this review we will consider the case of a wide Feshbach resonance. 


\section{Homonuclear diatomic molecules in Fermi gases}

\subsection{Weakly interacting gas of bosonic molecules. Molecule- molecule elastic interaction}

As we have shown in the previous section, the size of weakly bound bosonic molecules formed at a positive atom-atom scattering length $a$ in a two-species Fermi gas (region III in Fig. 1) is of the order of $a$. Therefore, at densities such that $n a^{3} \ll 1$, the atoms form a weakly interacting gas of these molecules. Moreover, under this condition at temperatures sufficiently lower than the molecular binding energy $\varepsilon_{0}$ and for equal concentrations of the two atomic components, practically all atoms are converted into molecules 52. This is definitely the case at temperatures below the temperature of quantum degeneracy $T_{d}=2 \pi \hbar^{2} n^{2 / 3} / M$ (the lowest one in the case of fermionic atoms with different masses, with $M$ being the mass of the heaviest atom). One can clearly see this by comparing $T_{d}$ with $\varepsilon_{0}$ given by Eq (5). Thus, one has a weakly interacting molecular Bose gas and the first question is related to the elastic interaction between the molecules.

For a weakly interacting gas the interaction energy in the system is equal to the sum of pair interactions and the energy per particle is $n g$ ( $2 n g$ for a non-condensed Bose gas), with $g$ being the coupling constant. In our case this coupling constant is given by $g=4 \pi \hbar^{2} a_{d d} /(M+m)$, where $a_{d d}$ is the scattering length for the molecule-molecule (dimer-dimer) elastic $s$-wave scattering, and $M, m$ are the masses of heavy and light atoms, respectively. The value of $a_{d d}$ is important for evaporative cooling of the molecular gas to the regime of BoseEinstein condensation and for the stability of the condensate. The Bose-Einstein condensate is stable for repulsive intermolecular interaction $\left(a_{d d}>0\right)$, and for $a_{d d}<0$ it collapses.

We thus see that for analyzing macroscopic properties of the molecular Bose gas one should first solve the problem of elastic interaction (scattering) between two molecules. In this section we present the exact solution of this problem for homonuclear molecules formed by fermionic atoms of different components (different internal states) in a two-component Fermi gas. The case of $M \neq$ $m$ will be discussed in Section 3. The solution for $M=m$ was obtained in Refs. 48, 49] assuming that the atom-atom scattering length $a$ greatly exceeds the characteristic radius of interatomic potential:

$$
a \gg R_{e} .
$$

Then, as in the case of the 3-body problem with fermions [55, 56, 53, 54, the amplitude of elastic interaction is determined only by $a$ and can be found in the zero-range approximation for the interatomic potential.

This approach was introduced in the two-body physics by Bethe and Peierls 57. The leading idea is to solve the equation for the free relative motion of two particles placing a boundary condition on the wavefunction $\psi$ at a vanishing 


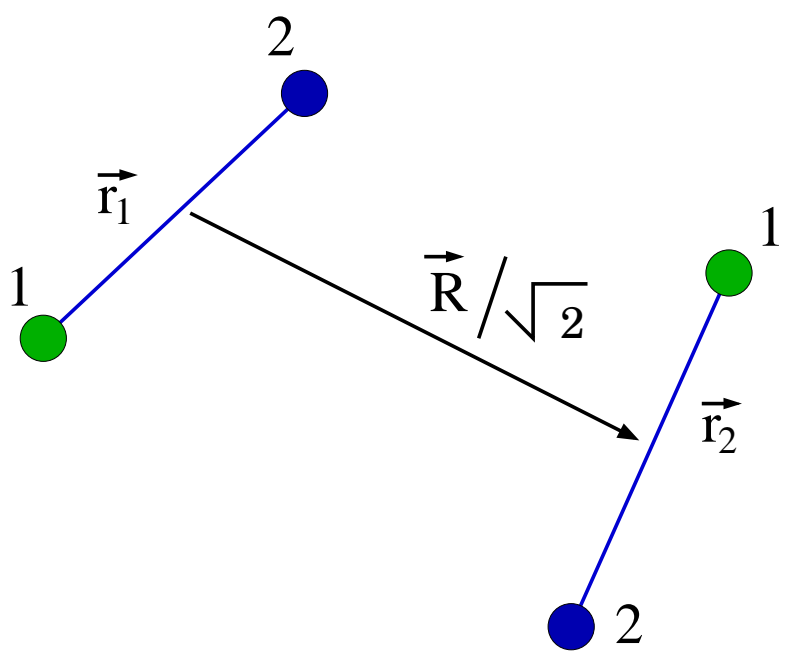

Figure 2: Set of coordinates for the four-body problem.

interparticle distance $r$ :

$$
\frac{(r \psi)^{\prime}}{r \psi}=-\frac{1}{a}, \quad r \rightarrow 0,
$$

which can also be rewritten as

$$
\psi \propto(1 / r-1 / a), \quad r \rightarrow 0 .
$$

One then gets the correct expression for the wavefunction at distances $r \gg R_{e}$. when $a \gg R_{e}$, Eq. (8) correctly describes the wavefunction of weakly bound and continuum states even at distances much smaller than $a$.

We now use the Bethe-Peierls approach for the problem of elastic moleculemolecule (dimer-dimer) scattering which is a 4-body problem described by the Schrödinger equation

$\left\{-\frac{\hbar^{2}}{m}\left(\nabla_{\mathbf{r}_{1}}^{2}-\nabla_{\mathbf{r}_{2}}^{2}-\nabla_{\mathbf{R}}^{2}\right)+U\left(r_{1}\right)+U\left(r_{2}\right)+\sum_{ \pm} U\left[\left(\mathbf{r}_{1}+\mathbf{r}_{2} \pm \sqrt{2} \mathbf{R}\right) / 2\right]-E\right\} \Psi=0$

where $m$ is the atom mass. Labeling fermionic atoms in different internal states by the symbols $\uparrow$ and $\downarrow$, the distance between two given $\uparrow$ and $\downarrow$ fermions is $\mathbf{r}_{1}$, and $\mathbf{r}_{2}$ is the distance between the other two. The distance between the centers of mass of these pairs is $\mathbf{R} / \sqrt{2}$, and $\left(\mathbf{r}_{1}+\mathbf{r}_{2} \pm \sqrt{2} \mathbf{R}\right) / 2$ are the separations between $\uparrow$ and $\downarrow$ fermions in the other two possible $\uparrow \downarrow$ pairs (see Fig. 2). The total energy is $E=-2 \varepsilon_{0}+\varepsilon$, with $\varepsilon$ being the collision energy, and $\varepsilon_{0}=$ $-\hbar^{2} / m a^{2}$ the binding energy of a dimer. The wavefunction $\Psi$ is symmetric with respect to the permutation of bosonic $\uparrow \downarrow$ pairs and antisymmetric with respect to permutations of identical fermions:

$$
\Psi\left(\mathbf{r}_{1}, \mathbf{r}_{2}, \mathbf{R}\right)=\Psi\left(\mathbf{r}_{2}, \mathbf{r}_{1},-\mathbf{R}\right)=-\Psi\left(\frac{\mathbf{r}_{1}+\mathbf{r}_{2} \pm \sqrt{2} \mathbf{R}}{2}, \frac{\mathbf{r}_{1}+\mathbf{r}_{2} \mp \sqrt{2} \mathbf{R}}{2}, \pm \frac{\mathbf{r}_{1}-\mathbf{r}_{2}}{\sqrt{2}}\right)(10)
$$


For the weak binding of atoms in the molecule assuming that the 2-body scattering length satisfies the inequality (6), at all interatomic distances (even much smaller than $a$ ) except for very short separations of the order of or smaller than $R_{e}$, the motion of atoms in the 4-body system is described by the freeparticle Schrödinger equation

$$
-\left[\nabla_{\mathbf{r}_{1}}^{2}+\nabla_{\mathbf{r}_{2}}^{2}+\nabla_{\mathbf{R}}^{2}+\frac{m E}{\hbar^{2}}\right] \Psi=0 .
$$

The correct description of this motion requires the 4-body wavefunction $\Psi$ to satisfy the Bethe-Peierls boundary condition for the vanishing distance in any pair of $\uparrow$ and $\downarrow$ fermions, i.e. for $\mathbf{r}_{1} \rightarrow 0, \mathbf{r}_{2} \rightarrow 0$, and $\mathbf{r}_{1}+\mathbf{r}_{2} \pm \sqrt{2} \mathbf{R} \rightarrow 0$. Due to the symmetry condition (10) it is necessary to require a proper behavior of $\Psi$ only at one of these boundaries. For $\mathbf{r}_{1} \rightarrow 0$ the boundary condition reads:

$$
\Psi\left(\mathbf{r}_{1}, \mathbf{r}_{2}, \mathbf{R}\right) \rightarrow f\left(\mathbf{r}_{2}, \mathbf{R}\right)\left(1 / 4 \pi r_{1}-1 / 4 \pi a\right) .
$$

The function $f\left(\mathbf{r}_{2}, \mathbf{R}\right)$ contains the information about the second pair of particles when the first two are on top of each other.

In the ultracold limit, where

$$
k a \ll 1,
$$

the molecule-molecule scattering is dominated by the contribution of the $s$-wave channel. The inequality (13) is equivalent to $\varepsilon \ll \varepsilon_{0}$ and, hence, the $s$-wave scattering can be analyzed from the solution of Eq. (11) with $E=-2 \varepsilon_{0}<0$. For large $R$ the corresponding wavefunction takes the form

$$
\Psi \approx \phi_{0}\left(r_{1}\right) \phi_{0}\left(r_{2}\right)\left(1-\sqrt{2} a_{d d} / R\right) ; \quad R \gg a,
$$

where the wavefunction of a weakly bound molecule is given by

$$
\phi_{0}(r)=\frac{1}{\sqrt{2 \pi a} r} \exp (-r / a)
$$

Combining Eqs. (12) and (14) we obtain the asymptotic expression for $f$ at large distances $R$ :

$$
f\left(\mathbf{r}_{2}, \mathbf{R}\right) \approx\left(2 / r_{2} a\right) \exp \left(-r_{2} / a\right)\left(1-\sqrt{2} a_{d d} / R\right) ; \quad R \gg a .
$$

In the case of $s$-wave scattering the function $f$ depends only on three variables: the absolute values of $\mathbf{r}_{2}$ and $\mathbf{R}$, and the angle between them. We now derive and solve the equation for $f$. The value of the molecule-molecule scattering length $a_{d d}$ is then deduced from the behavior of $f$ at large $R$ determined by Eq. (16).

We first establish a general form of the wavefunction $\Psi$ satisfying Eq. (11), with the boundary condition (12) and symmetry relations (10). In our case the total energy $E=-2 \hbar^{2} / m a^{2}<0$, and the Green function of Eq. (11) reads

$$
G(X)=(2 \pi)^{-9 / 2}(X a / \sqrt{2})^{-7 / 2} K_{7 / 2}(\sqrt{2} X / a),
$$


where $X=\left|S-S^{\prime}\right|, K_{7 / 2}(\sqrt{2} X / a)$ is the deacaying Bessel function, and $S=$ $\left\{\mathbf{r}_{1}, \mathbf{r}_{2}, \mathbf{R}\right\}$ is a 9-component vector. Accordingly, $\left|S-S^{\prime}\right|=\sqrt{\left(\mathbf{r}_{1}-\mathbf{r}_{1}{ }_{1}\right)^{2}+\left(\mathbf{r}_{2}-\mathbf{r}_{2}{ }_{2}\right)^{2}+\left(\mathbf{R}-\mathbf{R}^{\prime}\right)^{2}}$. The 4-body wavefunction $\Psi$ is regular everywhere except for vanishing distances between $\uparrow$ and $\downarrow$ fermions. Therefore, it can be expressed through $G\left(\left|S-S^{\prime}\right|\right)$ with coordinates $S^{\prime}$ corresponding to a vanishing distance between $\uparrow$ and $\downarrow$ fermions, i.e. for $\mathbf{r}_{1}^{\prime} \rightarrow 0, \mathbf{r}_{2}^{\prime} \rightarrow 0$, and $\left(\mathbf{r}^{\prime}{ }_{1}+\mathbf{r}^{\prime}{ }_{2} \pm \sqrt{2} \mathbf{R}^{\prime}\right) / 2 \rightarrow 0$. Thus, for the wavefunction $\Psi$ satisfying the symmetry relations (10) we have

$$
\begin{aligned}
& \Psi(S)=\Psi_{0}+\int d^{3} r^{\prime} d^{3} R^{\prime}\left[G\left(\left|S-S_{1}\right|\right)+G\left(\left|S-S_{2}\right|\right)\right. \\
& \left.-G\left(\left|S-S_{+}\right|\right)-G\left(\left|S-S_{-}\right|\right)\right] h\left(\mathbf{r}^{\prime}, \mathbf{R}^{\prime}\right)
\end{aligned}
$$

where $S_{1}=\left\{0, \mathbf{r}^{\prime}, \mathbf{R}^{\prime}\right\}, S_{2}=\left\{\mathbf{r}^{\prime}, 0,-\mathbf{R}^{\prime}\right\}$, and $S_{ \pm}=\left\{\mathbf{r}^{\prime} / 2 \pm \mathbf{R}^{\prime} / \sqrt{2}, \mathbf{r}^{\prime} / 2 \mp\right.$ $\left.\mathbf{R}^{\prime} / \sqrt{2} \mp \mathbf{r}^{\prime} \sqrt{2}\right\}$. The function $\Psi_{0}$ is a properly symmetrized finite solution of Eq. (11), regular at any distances between the atoms. For $E<0$, non-trivial solutions of this type do not exist and we have to put $\Psi_{0}=0$. The function $h\left(\mathbf{r}_{2}, \mathbf{R}\right)$ has to be determined by comparing $\Psi$ in Eq. (18) at $\mathbf{r}_{1} \rightarrow 0$, with the boundary condition (12).

Considering the limit $\mathbf{r}_{1} \rightarrow 0$ we extract the leading terms on the right hand side of Eq. (18). These are the terms that behave as $1 / r_{1}$ or remain finite in this limit. The last three terms in the square brackets in Eq. (18) provide a finite contribution

$$
\int d^{3} r^{\prime} d^{3} R^{\prime} h\left(\mathbf{r}^{\prime}, \mathbf{R}^{\prime}\right)\left[G\left(\left|\bar{S}_{2}-S_{2}\right|\right)-G\left(\left|\bar{S}_{2}-S_{+}\right|\right)-G\left(\left|\bar{S}_{2}-S_{-}\right|\right)\right],
$$

where $\bar{S}_{2}=\left\{0, \mathbf{r}_{2}, \mathbf{R}\right\}$. To find the contribution of the first term in the square brackets, we subtract and add an auxiliary quantity

$$
h\left(\mathbf{r}_{2}, \mathbf{R}\right) \int G\left(\left|S-S_{1}\right|\right) d^{3} r^{\prime} d^{3} R^{\prime}=\frac{h\left(\mathbf{r}_{2}, \mathbf{R}\right)}{4 \pi r_{1}} \exp \left(-\sqrt{2} r_{1} / a\right) .
$$

The result of the subtraction yields a finite contribution which for $r_{1} \rightarrow 0$ can be written as

$$
\begin{aligned}
& \int d^{3} r^{\prime} d^{3} R^{\prime}\left[h\left(\mathbf{r}^{\prime}, \mathbf{R}^{\prime}\right)-h\left(\mathbf{r}_{2}, \mathbf{R}\right)\right] G\left(\left|S-S_{1}\right|\right) \\
& =P \int d^{3} r^{\prime} d^{3} R^{\prime}\left[h\left(\mathbf{r}^{\prime}, \mathbf{R}^{\prime}\right)-h\left(\mathbf{r}_{2}, \mathbf{R}\right)\right] G\left(\left|\bar{S}_{2}-S_{1}\right|\right) ; \quad r_{1} \rightarrow 0,
\end{aligned}
$$

with the symbol $P$ denoting the principal value of the integral over $d r^{\prime}$ (or $d R^{\prime}$ ). A detailed derivation of Eq. (21) and the proof that the integral in the second line of this equation is convergent are given in Ref. [49.

In the limit $r_{1} \rightarrow 0$, the right hand side of Eq. (20) is equal to

$$
h\left(\mathbf{r}_{2}, \mathbf{R}\right)\left(1 / 4 \pi r_{1}-\sqrt{2} / 4 \pi a\right) .
$$


We thus find that for $\mathbf{r}_{1} \rightarrow 0$ the wavefunction $\Psi$ of Eq. (18) takes the form

$$
\Psi\left(\mathbf{r}_{1}, \mathbf{r}_{2}, \mathbf{R}\right)=\frac{h\left(\mathbf{r}_{2}, \mathbf{R}\right)}{4 \pi r_{1}}+\mathcal{R} ; \quad \mathbf{r}_{1} \rightarrow 0
$$

where $\mathcal{R}$ is the sum of regular $r_{1}$-independent terms given by Eqs. (19) and (21), and by the second term on the right hand side of Eq. (22). Equation (23) must coincide with Eq. (12), and comparing the singular terms of these equations we find $h\left(\mathbf{r}_{2}, \mathbf{R}\right)=f\left(\mathbf{r}_{2}, \mathbf{R}\right)$. As the quantity $\mathcal{R}$ must coincide with the regular term of Eq. (12), equal to $-f\left(\mathbf{r}_{2} \mathbf{R}\right) / 4 \pi a$, we obtain the following equation for the function $f$ :

$$
\begin{aligned}
& \int d^{3} r^{\prime} d^{3} R^{\prime}\left\{G\left(\left|\bar{S}-S_{1}\right|\right)\left[f\left(\mathbf{r}^{\prime}, \mathbf{R}^{\prime}\right)-f(\mathbf{r}, \mathbf{R})\right]+\left[G\left(\left|\bar{S}-S_{2}\right|\right)\right.\right. \\
& \left.\left.-\sum_{ \pm} G\left(\left|\bar{S}-S_{ \pm}\right|\right)\right] f\left(\mathbf{r}^{\prime}, \mathbf{R}^{\prime}\right)\right\}=(\sqrt{2}-1) f(\mathbf{r}, \mathbf{R}) / 4 \pi a .
\end{aligned}
$$

Here $\bar{S}=\{0, \mathbf{r}, \mathbf{R}\}$, and we omitted the symbol of the principal value for the integral in the first line of Eq. (24).

As we have already mentioned above, for $s$-wave scattering the function $f(\mathbf{r}, \mathbf{R})$ depends only on the absolute values of $\mathbf{r}$ and $\mathbf{R}$ and on the angle between them. Thus, Eq. (24) is an integral equation for the function of three variables. In order to find the molecule-molecule scattering length, it is more convenient to transform Eq. (24) into an equation for the momentum-space function $f(\mathbf{k}, \mathbf{p})=\int d^{3} r d^{3} R f(\mathbf{r}, \mathbf{R}) \exp (i \mathbf{k} \cdot \mathbf{r} / a+i \mathbf{p} \cdot \mathbf{R} / \sqrt{2} a)$, which yields the following expression:

$$
\begin{aligned}
& \sum_{ \pm} \int \frac{f\left(\mathbf{k} \pm\left(\mathbf{p}^{\prime}-\mathbf{p}\right) / 2, \mathbf{p}^{\prime}\right) \mathrm{d}^{3} p^{\prime}}{2+p^{\prime 2} / 2+\left(\mathbf{k} \pm\left(\mathbf{p}^{\prime}-\mathbf{p}\right) / 2\right)^{2}+\left(\mathbf{k} \pm\left(\mathbf{p}^{\prime}+\mathbf{p}\right) / 2\right)^{2}} \\
& =\int \frac{f\left(\mathbf{k}^{\prime},-\mathbf{p}\right) \mathrm{d}^{3} k^{\prime}}{2+k^{\prime 2}+k^{2}+p^{2} / 2}-\frac{2 \pi^{2}\left(1+k^{2}+p^{2} / 2\right) f(\mathbf{k}, \mathbf{p})}{\sqrt{2+k^{2}+p^{2} / 2}+1} .
\end{aligned}
$$

By making the substitution $f(\mathbf{k}, \mathbf{p})=\left(\delta(\mathbf{p})+g(\mathbf{k}, \mathbf{p}) / p^{2}\right) /\left(1+k^{2}\right)$ we reduce Eq. (25) to an inhomogeneous equation for the function $g(\mathbf{k}, \mathbf{p})$ :

$$
\begin{aligned}
& \frac{1}{\left(1+k^{2}+p^{2} / 4\right)^{2}-(\mathbf{k p})^{2}}+\frac{2 \pi^{2}\left(1+k^{2}+p^{2} / 2\right) g(\mathbf{k}, \mathbf{p})}{p^{2}\left(1+k^{2}\right)\left(\sqrt{2+k^{2}+p^{2} / 2}+1\right)} \\
& =-\sum_{ \pm} \int \frac{g\left(\mathbf{k} \pm\left(\mathbf{p}^{\prime}-\mathbf{p}\right) / 2, \mathbf{p}^{\prime}\right) d^{3} p^{\prime}}{p^{\prime 2}\left(2+p^{\prime 2} / 2+\left(\mathbf{k} \pm\left(\mathbf{p}^{\prime}-\mathbf{p}\right) / 2\right)^{2}+\left(\mathbf{k} \pm\left(\mathbf{p}^{\prime}+\mathbf{p}\right) / 2\right)^{2}\right)\left(1+\left(\mathbf{k} \pm\left(\mathbf{p}^{\prime}-\mathbf{p}\right) / 2\right)^{2}\right)} \\
& +\int \frac{g\left(\mathbf{k}^{\prime},-\mathbf{p}\right) d^{3} k^{\prime}}{p^{2}\left(2+k^{\prime 2}+k^{2}+p^{2} / 2\right)\left(1+k^{\prime 2}\right)} .
\end{aligned}
$$

In the case of $s$-wave scattering the function $g(\mathbf{k}, \mathbf{p})$ depends on the absolute values of $\mathbf{k}$ and $\mathbf{p}$ and on the angle between these vectors. For $\mathbf{p} \rightarrow 0$ this function tends to a finite value independent of $\mathbf{k}$. As one can easily establish on the 
basis of Eq. (16) and the definition of $g(\mathbf{k}, \mathbf{p})$, the molecule-molecule scattering length is given by $a_{d d}=-2 \pi^{2} a \lim _{\mathbf{p} \rightarrow 0} g(\mathbf{k}, \mathbf{p})$. Numerical calculations from Eq. (26) give with $2 \%$ accuracy [65]:

$$
a_{d d}=0.6 a>0 .
$$

This result was first obtained in Refs. 48, 49, from the direct numerical solution of Eq. (24) by fitting the obtained $f(\mathbf{r}, \mathbf{R})$ with the asymptotic form (16) at large $R$. The calculations show the absence of 4 -body weakly bound states, and the behavior of $f$ at small $R$ suggests a soft-core repulsion between dimers, with a range $\sim a$.

The result of Eq. (27) is exact, and it indicates the stability of molecular BEC with respect to collapse. Compared to earlier studies which assumed $a_{d d}=2 a$ 36, 58, Eq. (27) gives almost twice as small a sound velocity of the molecular condensate and a rate of elastic collisions smaller by an order of magnitude. The result of Eq. (27) has been confirmed by Monte Carlo calculations [59] and by calculations within the diagrammatic approach [60, 61. An approximate diagrammatic approach leading to $a=0.75 a$ has been developed in Ref. [58].

\subsection{Suppression of collisional relaxation}

Weakly bound dimers that we are considering are diatomic molecules in the highest ro-vibrational state (see Fig. 3). They can undergo relaxation into deeply bound states in their collisions with each other: for example, one of the colliding molecules may relax to a deeply bound state while the other one dissociates 62. The released energy is the binding energy of the final deep state, which is of the order of $\hbar^{2} / m R_{e}^{2}$. It is transformed into the kinetic energy of the particles in the outgoing collision channel and they escape from the trapped sample. Therefore, the process of collisional relaxation of weakly bound molecules determines the lifetime of a gas of these molecules and possibilities to Bose-condense this gas.

We now show that collisional relaxation is suppressed due to Fermi statistics for atoms in combination with a large size of weakly bound molecules [48, 49. The binding energy of the molecules is $\varepsilon_{0}=\hbar^{2} / m a^{2}$ and their size is $\sim a \gg R_{e}$. The size of deeply bound states is of the order of $R_{e}$. Therefore, the relaxation process may occur when at least three fermionic atoms are at distances $\sim R_{e}$ with respect to each other. As two of them are necessarily identical, due to the Pauli exclusion principle the relaxation probability acquires a small factor proportional to a power of $\left(q R_{e}\right)$, where $q \sim 1 / a$ is a characteristic momentum of the atoms in the weakly bound molecular state.

Relying on the inequality $a \gg R_{e}$ we outline a method that allows one to establish the dependence of the relaxation rate on the scattering length $a$, without going into a detailed analysis of the short-range behavior of the system. It is assumed that the amplitude of the inelastic relaxation process is much smaller than the amplitude of elastic scattering. Then the dependence of the relaxation rate on $a$ is related only to the $a$-dependence of the initial-state 4body wavefunction $\Psi$. We again consider the ultracold limit described by the 


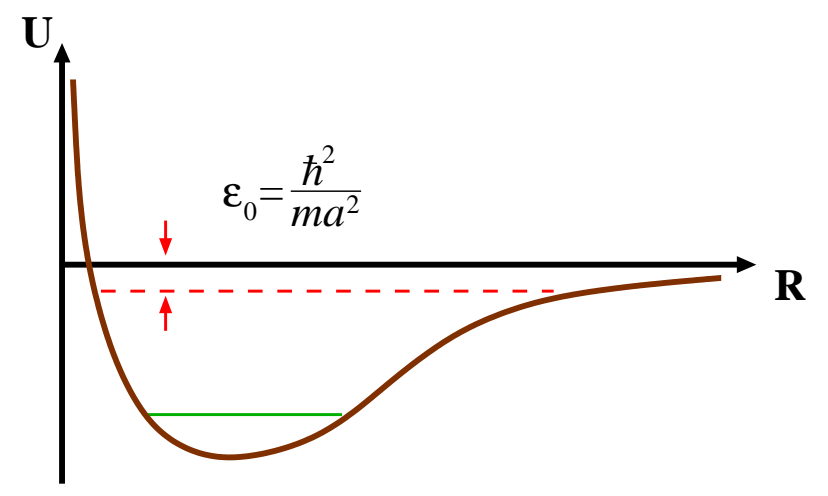

Figure 3: Interaction potential $U$ as a function of the distance $R$ between two distinguishable fermionic atoms. The dashed line shows the energy level of the weakly bound molecule, and the solid line the energy level of a deeply bound state.

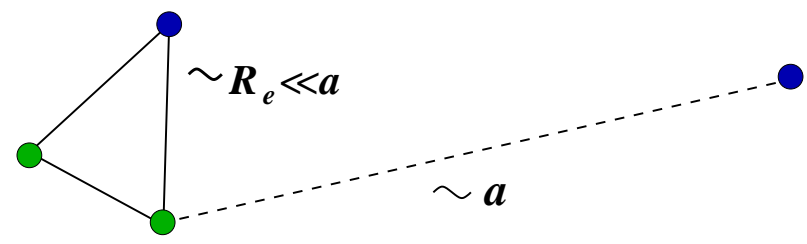

Figure 4: Configuration space contributing to the relaxation probability.

condition (13), where the relaxation process is dominated by the contribution of the $s$-wave molecule-molecule scattering.

The key point is that the relaxation process requires only three atoms to approach each other to short distances of the order of $R_{e}$. The fourth particle can be far away from these three and, in this respect, does not participate in the relaxation process. This distance is of the order of the size of a molecule, which is $\sim a \gg R_{e}$. We thus see that the configuration space contributing to the relaxation probability can be viewed as a system of three atoms at short distances $\sim R_{e}$ from each other and a fourth atom separated from this system by a large distance $\sim a$ (see Fig. 4). In this case the 4-body wavefunction decomposes into a product:

$$
\Psi=\eta(\mathbf{z}) \Psi^{(3)}(\rho, \Omega)
$$

where $\Psi^{(3)}$ is the wavefunction of the 3 -fermion system, $\rho$ and $\Omega$ are the hyperradius and the set of hyperangles for these fermions, $\mathbf{z}$ is the distance between their center of mass and the fourth atom. The wavefunction $\eta(\mathbf{z})$ describes the motion of this atom and is normalized to unity. Note that Eq. (28) remains valid for any hyperradius $\rho \ll|\mathbf{z}| \sim a$.

The transition to a deeply bound 2-body state occurs in the system of three 
atoms and does not change the wavefunction of the fourth atom, $\eta(\mathbf{z})$. Therefore, averaging the transition probability over the motion of the fourth particle, the rate constant for inelastic relaxation in dimer-dimer collisions can be written as

$$
\alpha_{r e l}=\alpha^{(3)} \int|\eta(\mathbf{z})|^{2} d^{3} z=\alpha^{(3)},
$$

where $\alpha^{(3)}$ is the rate constant for relaxation in the 3-atom system.

At interatomic distances $\sim R_{e}$ where the relaxation occurs, as well as at all distances where the hyperradius $\rho \ll a$, the wavefunction $\Psi^{(3)}$ is determined by the Schrödinger equation with zero energy and, hence, depends on the scattering length $a$ only through a normalization coefficient:

$$
\Psi^{(3)}=A(a) \psi ; \quad \rho \ll a,
$$

where the function $\psi$ is independent of $a$. The probability of relaxation and, hence, the relaxation rate constant are proportional to $|\Psi|^{2}$ at distances $\sim R_{e}$. We thus have

$$
\alpha_{r e l}=\alpha^{(3)} \propto|A(a)|^{2} .
$$

The goal then is to find the coefficient $A(a)$, which determines the dependence of the relaxation rate on $a$.

For this purpose it is sufficient to consider distances where $a \gg \rho \gg R_{e}$ and Eq. (30) is still valid. Then, using the zero-range approximation we find the coordinate dependence of the three-body wavefunction $\Psi^{(3)}$. The derivation is presented in Ref. [49] and the result is:

$$
\Psi^{(3)}=A(a) \Phi_{\nu}(\Omega) \rho^{\nu-1}, \quad \rho \ll a,
$$

where $\Phi_{\nu}(\Omega)$ is a normalized function of hyperangles, and the coefficient $\nu$ depends on the symmetry of $\Psi^{(3)}$. The $a$-dependence of the prefactor $A(a)$ can be determined from the following scaling arguments for the 4-body problem. The scattering length $a$ is the only length scale in our problem and we can measure all distances in units of $a$. Using two rescaled coordinates, $\rho=a \rho^{\prime}$ and $\mathbf{z}=a \mathbf{z}^{\prime}$, we see that $\Psi^{(3)}$ in Eqs. (32) and (28) becomes a function of $\rho / a$, multiplied by $A(a) a^{\nu-1}$. The wavefunction $\eta(\mathbf{z})$ is normalized to unity and hence it is a function of $\mathbf{z} / a$, multiplied by $a^{-3 / 2}$. Accordingly, the 4-body wavefunction $\Psi$ of Eq. (28) is a function of rescaled coordinates, multiplied by the coefficient $A(a) a^{\nu-5 / 2}$. By applying the same rescaling to Eq. (14) and using Eq. (15) we see that the same coefficient should be proportional to $a^{-3}$. Therefore, $A(a) \propto a^{-\nu-1 / 2}$ and $\alpha_{r e l} \propto a^{-s}$, where $s=2 \nu+1$.

The strongest relaxation channel corresponds to the lowest value of $\nu$. It is achieved in the case of $p$-wave symmetry in the three-body system described by the wavefunction $\Psi^{(3)}$ and is equal to $\nu=0.773$, which leads to $s=2.55$. Assuming that the short-range physics is characterized by the length scale $R_{e}$ and the energy scale $\hbar^{2} / m R_{e}^{2}$ we can restore the dimensions and write:

$$
\alpha_{r e l}=C\left(\hbar R_{e} / m\right)\left(R_{e} / a\right)^{s} ; \quad s=2.55
$$


where the coefficient $C$ depends on a particular system and can not be obtained using the zero-range approximation.

Note that the $p$-wave symmetry in the three-body system corresponds to $p$-wave scattering of a fermionic atom of one of the molecules (referred to as the 3-rd fermion) on the other molecule. Then the 4-th particle also undergoes $p$-wave scattering on this molecule in such a way that the total orbital angular momentum of the molecule-molecule collision is equal to zero. Since the 3-rd and 4-th fermions are bound to each other in the molecular state with a size $\sim a$, the relative momentum of their collisions with the other molecule is $\sim 1 / a$ and such $p$-wave collisions are not at all suppressed. The relaxation channel corresponding to $s$-wave scattering of the 3 -rd fermion on the molecule leads to $\nu=1.1662$ and hence to the relaxation rate proportional to $a^{-3.33}$ as in the case of ultracold atom-molecule collisions [48, 49]. Thus, for large $a$ this mechanism can be omitted. The channels, where the 3-rd fermion (and the 4-th one) scatters on the molecule with orbital angular momentum $l>1$, lead to even stronger decrease of the relaxation rate with increasing $a$ and hence can be neglected.

\subsection{Collisional stability and molecular BEC}

Equation (33) implies a remarkable collisional stability of weakly bound molecules consisting of fermionic atoms in two different internal states and a counterintuitive decrease of the relaxation rate with increasing the two-body scattering length $a$. For currently achieved values of the scattering length $a \sim 1000 \AA$, the suppression factor $\left(R_{e} / a\right)^{s}$ for the relaxation process is about 4 orders of magnitude. This effect is due to Fermi statistics for the atoms. It is not present for weakly bound molecules of bosonic atoms, even if they have the same large size. Indeed, as the size of weakly bound molecules is $\sim a$, identical fermionic atoms participating in the relaxation process have very small relative momenta $k \sim 1 / a$. Hence, the probability that they approach each other to short distances $\sim R_{e}$ where the relaxation transitions occur, should be suppressed as $\left(k R_{e}\right)^{2} \sim\left(R_{e} / a\right)^{2}$ compared to the case of molecules of bosonic atoms. The exponent $s$ in Eq. (33) is different from 2 due to the Frank-Condon factor for the relaxation transition and three-body dynamics.

The remarkable collisional stability of weakly bound molecules $\mathrm{K}_{2}$ and $\mathrm{Li}_{2}$ consisting of two fermionic atoms has been observed in experiments at JILA [12, 13, 14, Innsbruck [11, 15, 16, MIT [17, 18, ENS [10, 19], Rice 20], and Duke [21]. At molecular densities $n \sim 10^{13} \mathrm{~cm}^{-3}$ the lifetime of the gas ranges from tens of milliseconds to tens of seconds, depending on the value of the scattering length $a$. A strong decrease of the relaxation rate with increasing $a$, following from Eq. (33), is consistent with the experimental data. The potassium experiment at JILA [12] and lithium experiment at ENS [19] give the relaxation rate constant $\alpha_{r e l} \propto a^{-s}$, with $s \approx 2.3$ for $\mathrm{K}_{2}$, and $s \approx 1.9$ for $\mathrm{Li}_{2}$, in agreement with theory $(s \approx 2.55)$ within experimental uncertainty. The experimental and theoretical results for potassium and lithium are shown in Fig. 5 and Fig 6 . The absolute value of the rate constant for a ${ }^{6} \mathrm{Li}_{2}$ condensate is $\alpha_{\text {rel }} \approx 1 \times 10^{-13}$ 


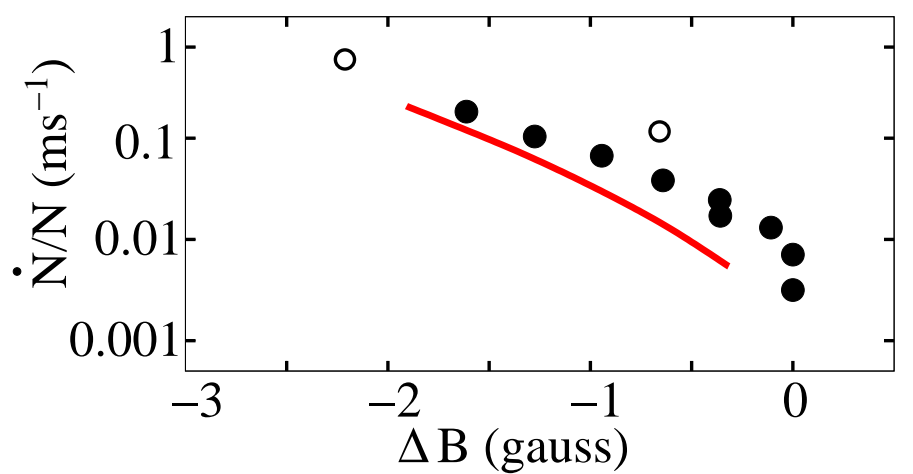

Figure 5: Two-body decay rate of a ${ }^{40} \mathrm{~K}_{2}$ ultracold molecular gas as a function of the magnetic field detuning from the ${ }^{40} \mathrm{~K}$ Feshbach resonance at $202 \mathrm{G}$. The dots indicate experimental values, and the solid line shows the theoretical results normalized to the experimental value at $\Delta B=-1.6 \mathrm{G}$.

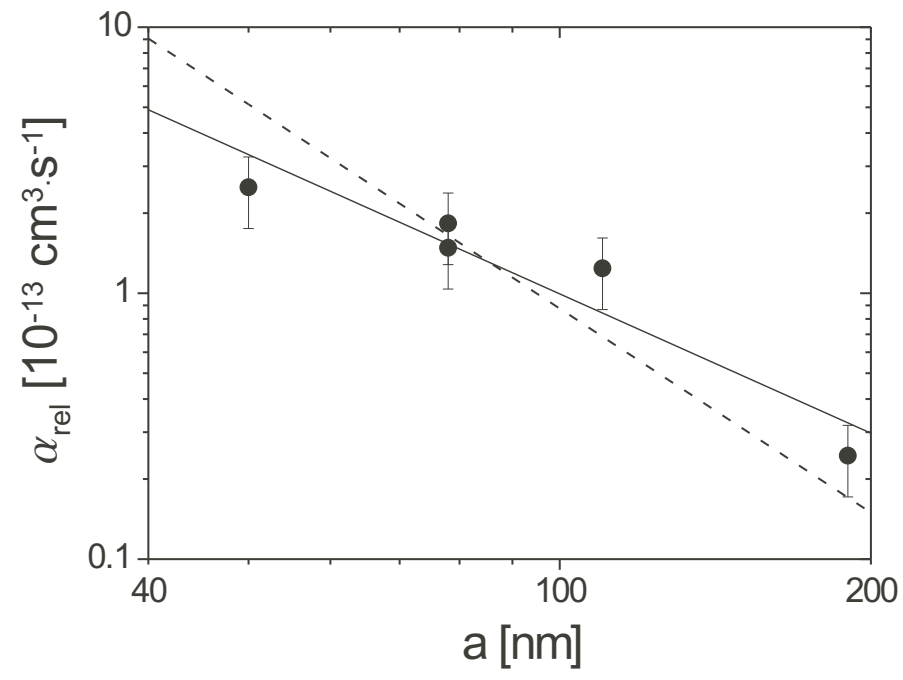

Figure 6: Two-body decay rate $\alpha_{\text {rel }}$ of a ${ }^{6} \mathrm{Li}_{2}$ molecular condensate as a function of interatomic scattering length near the ${ }^{6} \mathrm{Li}$ Feshbach resonance at $834 \mathrm{G}$. Solid line: least square fit, $\alpha_{r e l} \propto a^{-1.9 \pm 0.8}$. Dashed line, theory: $\alpha_{r e l} \propto a^{-2.55}$. The theoretical relaxation rate has been normalized to the experimental value at $a=78 \mathrm{~nm}$. 
$\mathrm{cm}^{3} / \mathrm{s}$ for the scattering length $a \approx 110 \mathrm{~nm}$. For $\mathrm{K}_{2}$ it is an order of magnitude higher at the same value of $a$ [12, which can be a consequence of a larger value of the characteristic radius of interaction $R_{e}$.

The suppression of the relaxation decay rate of weakly bound molecules of fermionic atoms has a crucial consequence for the physics of these molecules. At realistic temperatures the relaxation rate constant $\alpha_{r e l}$ is much smaller than the rate constant of elastic collisions $8 \pi a_{d d}^{2} v_{T}$, where $v_{T}$ is the thermal velocity. For example, for the $\mathrm{Li}_{2}$ weakly bound molecules at a temperature $T \sim 3 \mu \mathrm{K}$ and $a \sim 800 \AA$, the corresponding ratio is of the order of $10^{-4}$ or $10^{-5}$. This opens wide possibilities for reaching BEC of the molecules and cooling the Bosecondensed gas to temperatures of the order of its chemical potential. Long-lived BEC of weakly bound molecules has been observed for ${ }^{40} \mathrm{~K}_{2}$ at JILA [13, 14 . and for ${ }^{6} \mathrm{Li}_{2}$ at Innsbruck 15, 16, MIT [17, 18, ENS [19, Rice [20, Duke 21, and recently at Melbourne [63] and Tokyo [64. Measurements of the moleculemolecule scattering length confirm the result $a_{d d}=0.6 a$ with the accuracy up to $30 \%$ [16, 19].

\section{Heteronuclear molecules in Fermi-Fermi mix- tures}

\subsection{Effect of mass ratio on elastic intermolecular interac- tion}

We now focus on novel physics of heteronuclear molecules which are expected to be formed in a mixture of two different fermionic atoms (Fermi-Fermi mixture) at a large positive 2-body scattering length $a$. In several aspects, the physics is similar to that discussed above for homonuclear molecules of fermionic atoms in different internal states. However, for a large mass ratio of the atoms, the situation changes drastically. This is related to the existence of 3-body bound Efimov states, which in general makes it impossible to describe molecule-molecule scattering using only the value of the 2-body scattering length $a$.

We start with calculating the amplitude of elastic interaction (scattering) between weakly bound heteronuclear molecules consisting of a heavy (mass $M$ ) and light (mass $m$ ) fermionic atoms, assuming that the atom-atom scattering length satisfies the inequality $a \gg R_{e}$ and again considering the ultracold limit determined by the condition (13). In this case the scattering is dominated by the contribution of the $s$-wave channel, and we present here the exact results obtained in Ref. 65] using the zero-range approximation. Under the condition $k a \ll 1$ the collision energy is much smaller than the molecular binding energy $\varepsilon_{0}$. Hence, the $s$-wave molecule-molecule elastic scattering can be analyzed after setting the total energy equal to $-2 \varepsilon_{0}=-\hbar^{2} / \mu a^{2}$. In the zero-range approximation one should solve the four-body free-particle Schrödinger equation which again can be written in the form (11):

$$
\left[-\nabla_{\mathbf{r}_{1}}^{2}-\nabla_{\mathbf{r}_{2}}^{2}-\nabla_{\mathbf{R}}^{2}+2 / a^{2}\right] \Psi=0
$$




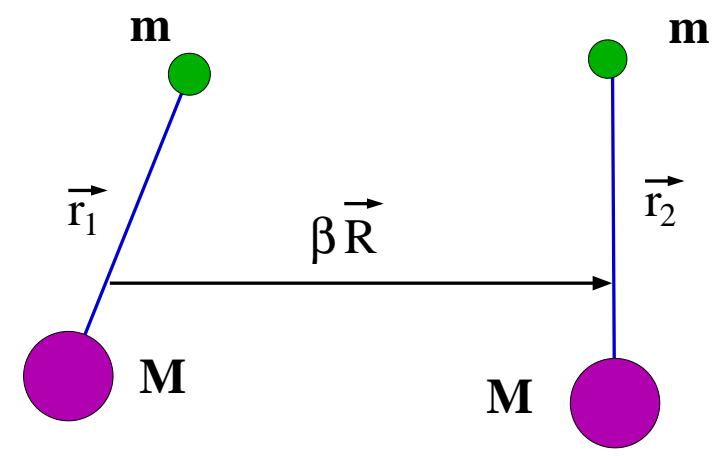

Figure 7: Set of coordinates for the four-body problem with two heteronuclear molecules.

where $\mathbf{r}_{1}$ is the distance between two given heavy and light fermions, and $\mathbf{r}_{2}$ the distance between the other two (see Fig. 7). However, it is now more convenient to define the distance between the centers of mass of these pairs as $\beta \mathbf{R}$, and the separations between the heavy and light fermions in the other two possible heavy-light pairs as $\mathbf{r}_{ \pm}=\alpha_{ \pm} \mathbf{r}_{1}+\alpha_{\mp} \mathbf{r}_{2} \pm \beta \mathbf{R}$, with $\beta=\sqrt{2 \alpha_{+} \alpha_{-}}, \alpha_{+}=\mu / M$, $\alpha_{-}=\mu / m$, and $\mu=m M /(m+M)$ being the reduced mass.

The symmetry condition (10) then takes the form:

$$
\Psi\left(\mathbf{r}_{1}, \mathbf{r}_{2}, \mathbf{R}\right)=\Psi\left(\mathbf{r}_{2}, \mathbf{r}_{1},-\mathbf{R}\right)=-\Psi\left(\mathbf{r}_{ \pm}, \mathbf{r}_{\mp}, \pm \beta\left(\mathbf{r}_{1}-\mathbf{r}_{2}\right) \mp\left(\alpha_{+}-\alpha_{-}\right) \mathbf{R}\right),
$$

and the Bethe-Peierls boundary condition should be applied for a vanishing distance in any pair of heavy and light fermions, i.e. for $\mathbf{r}_{1} \rightarrow 0, \mathbf{r}_{2} \rightarrow 0$, and $\mathbf{r}_{ \pm} \rightarrow 0$. For $\mathbf{r}_{1} \rightarrow 0$ it is again given by Eq. (12).

Due to the change in the definition of the coordinates the asymptotic expression for the wavefunction $\Psi$ at large distances $R$ now reads:

$$
\Psi \approx \phi_{0}\left(r_{1}\right) \phi_{0}\left(r_{2}\right)\left(1-a_{d d} / \beta R\right) ; \quad R \gg a,
$$

where the notation $a_{d d}$ is again used for the molecule-molecule scattering length, and the wavefunction of a weakly bound molecule is given by Eq. (15). Then the asymptotic expression for the function $f\left(\mathbf{r}_{2}, \mathbf{R}\right)$ at large $R$ is given by:

$$
f\left(\mathbf{r}_{2}, \mathbf{R}\right) \approx\left(2 / r_{2} a\right) \exp \left(-r_{2} / a\right)\left(1-a_{d d} / \beta R\right) ; \quad R \gg a
$$

For $s$-wave scattering the function $f$ depends only on three variables: the absolute values of $\mathbf{r}_{2}$ and $\mathbf{R}$, and the angle between them. Using the procedure described in Section 2 we obtain for $f$ the same integral equation (24). The effect of different masses is contained in the expressions for the vectors $S_{ \pm}$, which now read $S_{ \pm}=\left\{\alpha_{\mp} \mathbf{r}^{\prime} \pm \beta \mathbf{R}^{\prime}, \alpha_{ \pm} \mathbf{r}^{\prime} \mp \beta \mathbf{R}^{\prime}, \mp \beta \mathbf{r}^{\prime} \mp\left(\alpha_{+}-\alpha_{-}\right) \mathbf{R}^{\prime}\right\}$.

In order to find the molecule-molecule scattering length as a function of the mass ratio $M / m$ it is again more convenient to transform the integral equation for the function $f(\mathbf{r}, \mathbf{R})$ into an equation in the momentum space. Introducing 


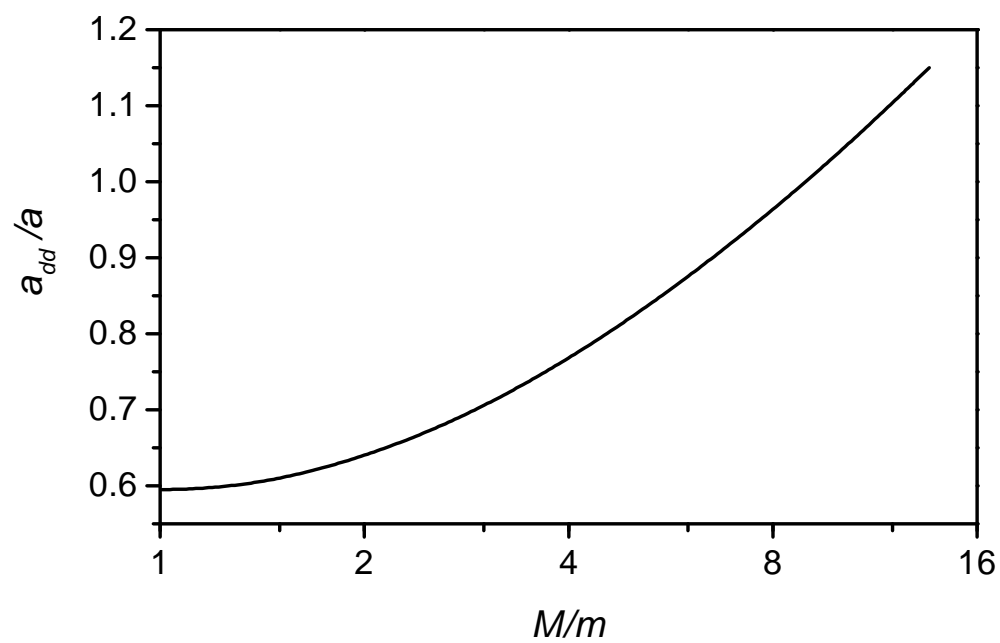

Figure 8: The ratio $a_{d d} / a$ versus $M / m$.

the Fourier transform of the function $f(\mathbf{r}, \mathbf{R})$ as $f(\mathbf{k}, \mathbf{p})=\int d^{3} r d^{3} R f(\mathbf{r}, \mathbf{R}) \exp (i \mathbf{k} \cdot \mathbf{r} / a+$ $i \beta \mathbf{p} \cdot \mathbf{R} / a)$, we obtain the following momentum-space equation:

$$
\begin{aligned}
& \sum_{ \pm} \int \frac{f\left(\mathbf{k} \pm \alpha_{\mp}\left(\mathbf{p}^{\prime}-\mathbf{p}\right), \mathbf{p}^{\prime}\right) \mathrm{d}^{3} p^{\prime}}{2+\beta^{2} p^{\prime 2}+\left(\mathbf{k} \pm \alpha_{\mp}\left(\mathbf{p}^{\prime}-\mathbf{p}\right)\right)^{2}+\left(\mathbf{k} \pm \alpha_{ \pm}\left(\mathbf{p}^{\prime}+\mathbf{p}\right)\right)^{2}} \\
& =\int \frac{f\left(\mathbf{k}^{\prime},-\mathbf{p}\right) \mathrm{d}^{3} k^{\prime}}{2+k^{\prime 2}+k^{2}+\beta^{2} p^{2}}-\frac{2 \pi^{2}\left(1+k^{2}+\beta^{2} p^{2}\right) f(\mathbf{k}, \mathbf{p})}{\sqrt{2+k^{2}+\beta^{2} p^{2}}+1} .
\end{aligned}
$$

By making the substitution $f(\mathbf{k}, \mathbf{p})=\left(\delta(\mathbf{p})+g(\mathbf{k}, \mathbf{p}) / p^{2}\right) /\left(1+k^{2}\right)$ we reduce Eq. (37) to an inhomogeneous equation for the function $g(\mathbf{k}, \mathbf{p})$. This equation is similar to Eq. (26) and we do not present it here because of its complexity. As well as in the case of $M=m$, for $\mathbf{p} \rightarrow 0$ the function $g(\mathbf{k}, \mathbf{p})$ tends to a finite value independent of $\mathbf{k}$. The molecule-molecule scattering length is again given by $a_{d d}=-2 \pi^{2} a \lim _{\mathbf{p} \rightarrow 0} g(\mathbf{k}, \mathbf{p})$. In Fig. 7 we display the ratio $a_{d d} / a$ versus the mass ratio $M / m$, found in Ref. [65. For the case of homonuclear molecules $(m=M)$ we recover the molecule-molecule scattering length $a_{d d}=0.6 a$.

The universal dependence of $a_{d d} / a$ on the mass ratio, presented in Fig. 8, can be established in the zero-range approximation only if $M / m$ is smaller than 13.6. Calculations then show the absence of four-body weakly bound states, and for $M / m \sim 1$ the behavior of $f$ suggests a soft-core repulsion between molecules, with a range $\sim a$. For the mass ratio larger than the limiting value 13.6, the description of the molecule-molecule scattering requires a three-body parameter coming from the short-range behavior of the three-body subsystem consisting of one light and two heavy fermions $[53,54$. A qualitative explanation of this behavior will be given in subsection 3.3 . 


\subsection{Collisional relaxation for moderate mass ratios}

The most exciting physics with weakly bound (heteronuclear) bosonic molecules consisting of different fermionic atoms is related to their collisional stability. As well as homonuclear molecules discussed above, they are in the highest rovibrational state and hence undergo relaxation into deeply bound states in moleculemolecule collisions, which leads to decay of the sample. The collisional relaxation determines the lifetime of the Bose gas of weakly bound molecules and there is a subtle question of whether and how the mass ratio $M / m$ can influence the suppression of this process 65, originating from the Fermi statistics for the atoms and playing a crucial role in the case of homonuclear molecules. In a similar way, behaving themselves as point-like bosons at large intermolecular distances, heteronuclear molecules "remember" that they consist of fermions when the intermolecular separation becomes smaller than the molecule size $(\sim a)$. The relaxation requires the presence of at least three fermions at separations $\sim R_{e}$ from each other. Two of them are necessarily identical, so that due to the Pauli exclusion principle the relaxation probability acquires a small factor proportional to a power of $\left(q R_{e}\right)$, where $q \sim 1 / a$ is a characteristic momentum of the atoms in the weakly bound molecular state. What changes in this picture when the fermionic atoms forming the molecule have different masses?

We first consider molecule-molecule relaxation collisions for the case where the mass ratio is smaller than the limiting value 13.6 and short-range physics is not supposed to influence the dependence of the relaxation rate on the 2-body scattering length $a$. As well as in the case of homonuclear molecules in Section 2, we assume the inequality $a \gg R_{e}$ and consider the ultracold limit described by Eq. (13). The configuration space contributing to the relaxation probability can be again viewed as a system of only three atoms at short distances $~$ $R_{e}$ from each other and a fourth atom separated from this system by a large distance $\sim a$. Hence, the four-body wavefunction decomposes into a product according to Eq. (28): $\Psi=\eta(\mathbf{z}) \Psi^{(3)}(\rho, \Omega)$, with $\Psi^{(3)}$ being the wavefunction of the three-fermion system, and $\rho \ll a$ and $\Omega$ being the hyperradius and the set of hyperangles for these fermions. The distance between their center of mass and the fourth atom is $\mathbf{z}$, and the function $\eta(\mathbf{z})$ describes the motion of this atom. In the case of fermionic atoms with different masses there are two possible choices of a three-body subsystem out of four fermions. The most important is the relaxation in the system of one atom with the mass $m$ and two heavier atoms with masses $M$.

We then use the same arguments as in Section 2 and obtain equation (32) for the function $\Psi^{(3)}$ at distances where $R_{e} \ll \rho \ll a$ : $\Psi^{(3)}=A(a) \Phi_{\nu}(\Omega) \rho^{\nu-1}$, with the coefficient $A(a)$ determining the $a$-dependence of the relaxation rate according to Eq. (31): $\alpha_{r e l}=\alpha^{(3)} \propto|A(a)|^{2}$. A similar scaling procedure as in Section 2 again leads to $\alpha_{r e l} \propto a^{-s}$, where $s=2 \nu+1$, and restoring the dimensions we can write the relaxation rate in the form (33): $\alpha_{r e l}=C\left(\hbar R_{e} / m\right)\left(R_{e} / a\right)^{s}$, with a coefficient $C$ depending on the mass ratio and on short-range physics.

However, the exponent $s$ now depends not only on the symmetry of the three-body wave function $\Psi^{(3)}$, but also on the mass ratio $M / m$. The smallest 


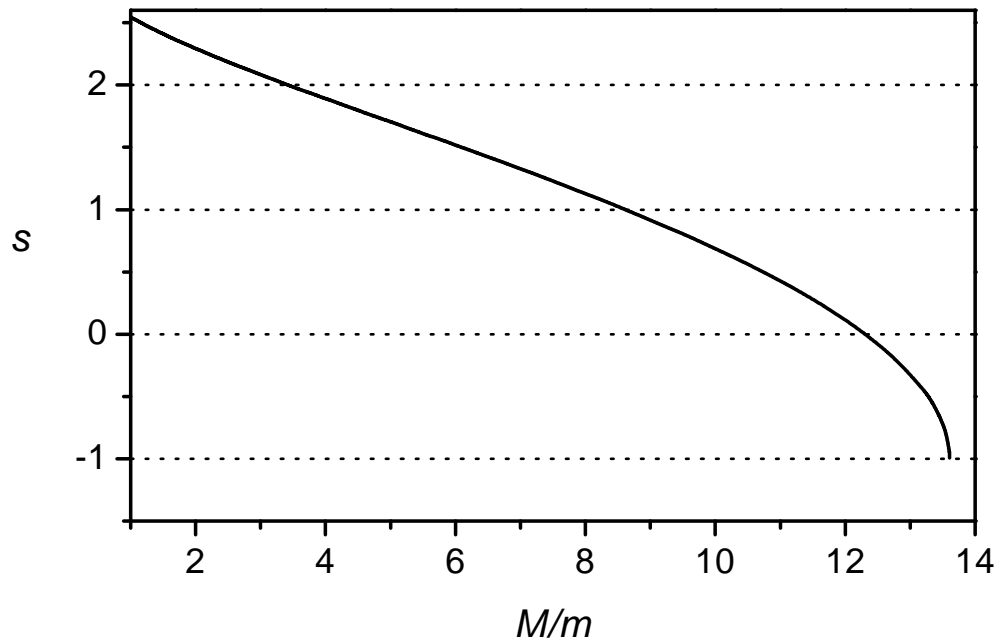

Figure 9: The dependence of the exponent $s=2 \nu+1$ in Eq. (33) on the mass ratio $M / m$.

value of $\nu$, i.e. the one corresponding to the leading relaxation channel at large $a$, is achieved for the $p$-wave symmetry in the system of one light and two heavy fermions [54. In the interval $-1 \leq \nu<2$ it is given by the root of the function [54]:

$$
\lambda(\nu)=\frac{\nu(\nu+2)}{\nu+1} \cot \frac{\pi \nu}{2}+\frac{\nu \sin \gamma \cos (\nu \gamma+\gamma)-\sin (\nu \gamma)}{(\nu+1) \sin ^{2} \gamma \cos \gamma \sin (\pi \nu / 2)}
$$

where $\gamma=\arcsin [M /(M+m)]$. A detailed derivation of Eq. (38) is given in Ref. [54.

In the case of equal masses we recover $s=2 \nu+1 \approx 2.55$ obtained in Section 2, and it slowly decreases with increasing the mass ratio (see Fig. 9). For $M / m \sim 1$ nothing dramatic happens: the suppression of the relaxation rate with increasing the 2-body scattering length a becomes slightly weaker than for homonuclear molecules. However, for the mass ratio approaching the limiting value 13.6 the exponent $s$ first reaches zero and then becomes negative, showing even an increase of the relaxation rate with $a$. We will give a qualitative explanation of this phenomenon using the Born-Oppenheimer approximation for the system of two heavy and one light atom.

\subsection{Born-Oppenheimer picture of collisional relaxation}

In the Born-Oppenheimer approximation one assumes that the state of a fast light atom adiabatically adjusts itself to the positions $\mathbf{R}_{1}$ and $\mathbf{R}_{2}$ of the slow heavy atoms. One then finds the wavefunction and energy of a bound state of the light atom with two heavy atoms at a given separation between them, $\mathbf{R}=\mathbf{R}_{1}-\mathbf{R}_{2}$. For convenience, from this point on we change the notations and 
use $\mathbf{R}$ to specify the distance between the heavy atoms, and $\mathbf{r}$ the coordinate of a light atom relative to their center of mass. In general, there are two states of a light atom in the field of two heavy ones: the gerade state $(+)$ with the wavefunction remaining unchanged under permutation of the heavy atoms $(\mathbf{R} \rightarrow$ $-\mathbf{R})$, and the ungerade state $(-)$ with the wavefunction changing its sign under this operation. The corresponding wavefunctions are given by

$$
\psi_{\mathbf{R}}^{ \pm}(\mathbf{r})=\mathcal{N}_{ \pm}\left(\frac{e^{-\kappa_{ \pm}(R)|\mathbf{r}-\mathbf{R} / 2|}}{|\mathbf{r}-\mathbf{R} / 2|} \pm \frac{e^{-\kappa_{ \pm}(R)|\mathbf{r}+\mathbf{R} / 2|}}{|\mathbf{r}+\mathbf{R} / 2|}\right),
$$

where $\mathcal{N}_{ \pm}$are normalization coefficients which depend on $R$. The corresponding binding energies are

$$
\epsilon_{ \pm}(R)=-\hbar^{2} \kappa_{ \pm}^{2}(R) / 2 m
$$

and the parameters $\kappa_{ \pm}(R)$ follow from the equation

$$
\kappa_{ \pm}(R) \mp \exp \left[-\kappa_{ \pm}(R) R\right] / R=1 / a .
$$

Equation (41) is obtained by using the Bethe-Peierls boundary condition (8) for the wavefunctions $\psi_{\mathbf{R}}^{ \pm}$of Eq. (39) at vanishing light-heavy atom separations $|\mathbf{r} \pm \mathbf{R} / 2|$.

The ungerade (-) state energy is always higher than the energy of the gerade $(+)$ state. Moreover, for $R<a$ the ungerade state is no longer bound and we are dealing only with the gerade bound state. In the limit of $R \ll a$ Eq. (41) gives $\kappa_{+}=0.56$. Then the energy of the gerade bound state, representing an effective potential for the relative motion of the heavy atoms, is given by

$$
\epsilon_{+}(R)=-0.16 \hbar^{2} / m R^{2} .
$$

We thus see that when the heavy atoms are separated from each other by a distance $R \ll a$, the light atom mediates an effective $1 / R^{2}$ attraction between them. Actually, the same result follows from the Efimov picture of effective interaction in a three-body system [53] and the Born-Oppenheimer approximation only gives a physically transparent illustration of this picture 65, 66. For a large mass ratio the mediated attractive potential $\epsilon_{+}(R)=-0.16 \hbar^{2} / m R^{2}$ strongly modifies the physics of the relaxation process. It competes with the Pauli principle which in terms of effective interaction manifests itself in the centrifugal $1 / R^{2}$ repulsion between the heavy atoms. The presence of this repulsion is clearly seen from the fact that the light-atom wavefunction $\psi_{\mathbf{R}}^{+}(\mathbf{r})$ does not change sign under permutation of heavy fermions. As the total wavefunction of the three-body system $\psi_{\mathbf{R}}^{+}(\mathbf{r}) \chi(\mathbf{R})$ is antisymmetric with respect to this permutation, the wavefunction of the relative motion of heavy atoms $\chi(\mathbf{R})$ should change its sign. Therefore, $\chi(\mathbf{R})$ contains only partial waves with odd angular momenta, and for the lowest angular momentum ( $p$-wave) the centrifugal barrier is $U_{c}(R)=2 \hbar^{2} / M R^{2}$. For comparable masses it is significantly stronger than $\epsilon_{+}(R)$. Thus, we have the physical picture discussed in the case of homonuclear molecules: the Pauli principle (centrifugal barrier) reduces the probability for 
the atoms to be at short distances and, as a consequence, the relaxation rate decreases with increasing the atom-atom scattering length $a$.

The role of the effective attraction increases with $M / m$. As a result, the decrease of the relaxation rate with increasing $a$ becomes weaker. The exponent $s$ in Eq. (33) continuously decreases with increasing $M / m$ and becomes zero for $M / m=12.33$ (see Fig. 9). In the Born-Oppenheimer picture this means that at this point one has a balance between the mediated attraction and the centrifugal repulsion. A further increase in $M / m$ makes $s$ negative and it reaches the value $s=-1$ for the critical mass ratio $M / m=13.6$. Thus, in the range $12.33<M / m<13.6$ the relaxation rate increases with $a$.

For an overcritical mass ratio $M / m>13.6$ we have a well-known phenomenon of the fall of a particle to the center in an attractive $1 / R^{2}$ potential [25. In this case the shape of the wavefunction at distances of the order of $R_{e}$ can significantly influence the large-scale behavior and a short-range three-body parameter is required to describe the system. The wavefunction of heavy atoms $\chi(\mathbf{R})$ acquires many nodes at short distances $R$, which indicates the appearance of 3-body bound Efimov states.

\subsection{Molecules of heavy and light fermionic atoms}

The discussion of the previous subsection shows that weakly bound molecules of heavy and light fermions become collisionally unstable for the mass ratio $\mathrm{M} / \mathrm{m}$ close to the limiting value 13.6. The effect of the Pauli principle becomes weaker than the attraction between heavy atoms at distances $R \ll a$, mediated by light fermions. However, this picture explains only the dependence of the relaxation rate on the 2-body scattering length $a$. At the same time, for heteronuclear molecules the relaxation rate and the amplitude of elastic molecule-molecule interaction can also depend on the mass ratio irrespective of the value of $a$ and short-range physics. To elucidate this dependence, we will look at the interaction between the molecules of heavy and light fermions at large intermolecular separations.

We consider the interaction between two such molecules in the Born-Oppenheimer approximation and calculate the wavefunctions and binding energies of two light fermions in the field of two heavy atoms fixed at their positions $\mathbf{R}_{1}$ and $\mathbf{R}_{2}$. The sum of the corresponding binding energies gives an effective interaction potential $U_{\text {eff }}$ for the heavy fermions as a function of the separation $R=\left|\mathbf{R}_{1}-\mathbf{R}_{2}\right|$ between them.

For $R>a$, there are two bound states, gerade $(+)$ and ungerade $(-)$, for a light atom interacting with a pair of fixed heavy atoms. Their wavefunctions are given by Eq. (39), and the corresponding binding energies follow from Eqs. (40) and (41). For large $R$ satisfying the condition $\exp (-R / a) \ll 1$, Eq. (40) yields:

$$
\epsilon_{ \pm}(R) \approx-\left|\epsilon_{0}\right| \mp 2\left|\epsilon_{0}\right| \frac{a}{R} \exp (-R / a)+\frac{U_{\mathrm{ex}}(R)}{2},
$$

where the binding energy of a single molecule, $\epsilon_{0}$, is given by Eq. (5) with the 


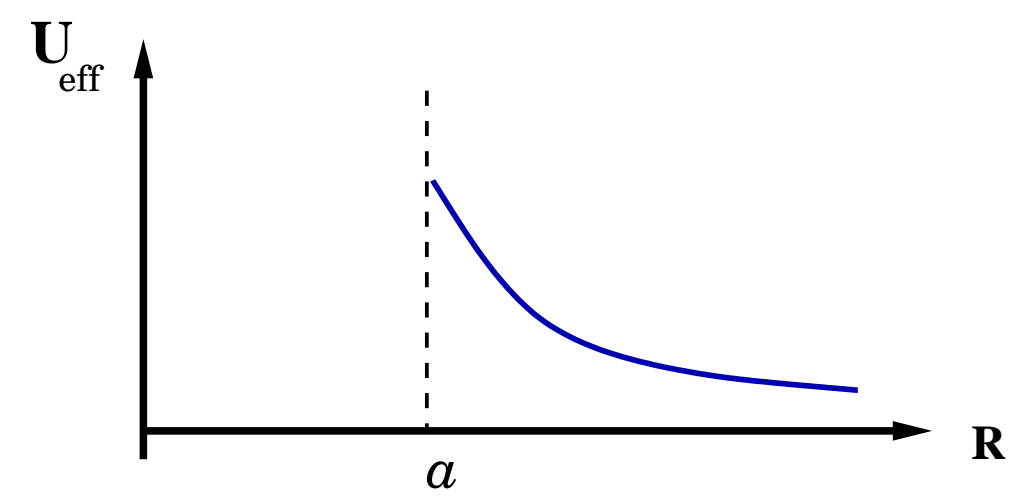

Figure 10: Interaction potential for two molecules of heavy and light fermions as a function of the separation $R$ between the heavy atoms.

reduced mass $\mu$ very close to the light atom mass $m$.

$$
U_{\text {ex }}(R)=4\left|\epsilon_{0}\right| \frac{a}{R}\left(1-\frac{a}{2 R}\right) \exp (-2 R / a) .
$$

Since the light fermions are identical, their two-body wavefunction is an antisymmetrized product of gerade and ungerade wavefunctions:

$$
\psi_{\mathbf{R}}\left(\mathbf{r}_{1}, \mathbf{r}_{\mathbf{2}}\right)=\left[\psi_{\mathbf{R}}^{+}\left(\mathbf{r}_{1}\right) \psi_{\mathbf{R}}^{-}\left(\mathbf{r}_{\mathbf{2}}\right)-\psi_{\mathbf{R}}^{+}\left(\mathbf{r}_{\mathbf{2}}\right) \psi_{\mathbf{R}}^{-}\left(\mathbf{r}_{1}\right)\right] / \sqrt{2} .
$$

The Born-Oppenheimer adiabatic approach is valid at distances $R>a$, where the effective interaction potential between the molecules, $U_{\text {eff }}$, is the sum of $\epsilon_{+}(R)$ and $\epsilon_{-}(R)$ (one should add $2\left|\epsilon_{0}\right|$ so that $U_{\text {eff }}(R) \rightarrow 0$ for $R \rightarrow \infty$ ). This potential is displayed in Fig 10.

At sufficiently large inter-heavy separations where Eq. (43) is valid, the effective potential can be written as

$$
U_{\mathrm{eff}}(R)=\epsilon_{+}(R)+\epsilon_{-}(R)+2\left|\epsilon_{0}\right| \approx U_{\mathrm{ex}}(R) .
$$

The potential $U_{\text {ex }}$ originates from the exchange of light fermions and thus can be treated as an exchange interaction. It is purely repulsive and, according to Eq. (44), has the asymptotic shape of a Yukawa potential at large $R$. Direct calculations show that $U_{\text {ex }}$ is a very good approximation to $U_{\text {eff }}$ for $R \gtrsim 1.5 a$.

We now demonstrate the calculation of the dimer-dimer scattering length $a_{d d}$ in the limit of $M / m \gg 1$ [67]. In the Born-Oppenheimer approach the Schrödinger equation for the relative motion of two molecules reads;

$$
\left(-\left(\hbar^{2} / m\right) \nabla_{\mathbf{R}}^{2}+U_{\mathrm{eff}}(R)-\epsilon\right) \Psi(\mathbf{R})=0
$$

where $\epsilon$ is the collision energy. Note, that the repulsive effective potential is inversely proportional to the light mass $m$, whereas the kinetic energy operator in Eq. (47) has a prefactor $1 / M$. Therefore, for a large mass ratio $M / m$, the heavy 
atoms approach each other at distances smaller than $a$ with an exponentially small tunneling probability $P \propto \exp (-B \sqrt{M / m})$, where $B \sim 1$. This leads to the relaxation rate constant

$$
\alpha_{r e l} \propto \exp (-B \sqrt{M / m}),
$$

which strongly decreases with increasing the mass ratio $M / m$.

The analysis shows that the elastic part of the scattering amplitude can be calculated with a very high accuracy using Eq. (47) for $M / m \gtrsim 20$ and is practically insensitive to the way we choose the boundary condition for the wavefunction at $R=a$. The dominant contribution to the scattering comes from distances in the vicinity of $R=a_{d d} \gg a$, where the effective potential can be approximated by Eq. (44) with a constant preexponential factor:

$$
U_{\text {eff }}(R) \approx 2 \hbar^{2}\left(m a a_{d d}\right)^{-1} \exp (-2 R / a) .
$$

Then, the zero energy solution of Eq. (47) that decays at smaller $R$, reads:

$$
\Psi(R)=\frac{a}{R} K_{0}\left(\sqrt{\frac{2 M}{m} \frac{a}{a_{d d}}} e^{-R / a}\right),
$$

where $K_{0}$ is the decaying Bessel function. Comparing the result of Eq. (50) at large $R$ with the asymptotic behavior $\Psi(R) \propto\left(1-a_{d d} / R\right)$ we obtain an equation for $a_{d d}$ :

$$
a_{d d}=\frac{a}{2} \ln \left(\frac{e^{2 \gamma}}{2} \frac{M}{m} \frac{a}{a_{d d}}\right) .
$$

This gives

$$
a_{d d} \approx a \ln \sqrt{M / m}
$$

and the scattering cross section is

$$
\sigma_{d d}=8 \pi a_{d d}^{2}
$$

From Eq. (50) we see that the interval of distances near $R=a_{d d}$, where the wavefunction changes, is of the order of $a$. This justifies the use of Eq. (49). In fact, the corrections to Eq. (51) can be obtained by treating the difference between Eqs. (44) and (49) perturbatively. In this way the first order correction to the dimer-dimer scattering length is $-(3 / 4) a^{2} / a_{d d}$, where $a_{d d}$ is determined from Eq. (51).

Qualitatively, $U_{\text {eff }}(R)$ can be viewed as a hard-core potential with the radius $a_{d d}$, where the edge is smeared out on a lengthscale $\sim a \ll a_{d d}$. Therefore, the ultracold limit for dimer-dimer collisions, required for the validity of Eq. (53), is realized for relative momenta of the dimers, $k$, satisfying the inequality

$$
k a_{d d} \ll 1 .
$$

It can be useful (see Ref. [68]) to approximate the potential $U_{\text {eff }}$ by a pure hard core with the radius $a_{d d}$. This approximation works under the condition $k a \ll 1$, which is less strict than Eq. (54). 
Let us now mention that numerical calculations Ref. 67] show that there are no resonances in the dimer-dimer scattering amplitude, which could appear in the presence of a weakly bound state of two dimers. Here we give a qualitative explanation of the absence of these bound states. Suppose there is such a state with energy $\epsilon \rightarrow 0$. Then, at distances $R>a$ the wavefunction of the heavy atoms should exponentially decay on the distance scale $\sim a \sqrt{m / M} \ll a$, since $U_{\text {eff }}$ represents a barrier with the height $\sim 1 / m a^{2}$. This means that the heavy atoms in such a bound state should be localized mostly at distances smaller than $a$. The gerade light atom is also localized at these distances as seen from the shape of the function $\psi^{+}$. The motion of the ungerade light atom relative to the localized trimer can be viewed as scattering with odd values of the angular momentum, and due to the centrifugal barrier the bound states of this atom with the trimer should be localized at distances $\sim a$ from the heavy atoms. In this case one would expect the BO approximation to work, since the ungerade light atom is moving much faster than the heavy atoms. However, this leads to a contradiction, because in the $\mathrm{BO}$ approach discussed above the ungerade state at interheavy separations $R<a$ is unbound. We thus conclude that weakly bound states of two dimers are absent.

Although there are no resonances in the dimer-dimer collisions, there are branch-cut singularities in the scattering amplitude. They are related to the presence of inelastic processes in molecule-molecule collisions. These represent the relaxation of one of the colliding dimers into a deeply bound state, the other dimer being dissociated, and the formation of bound trimers consisting of two heavy and one light atom, the other light atom carrying away the released binding energy.

\subsection{Trimer states}

The trimer states, which in most cases can be called Efimov trimers, are interesting objects. Their existence can be seen from the BO picture for two heavy atoms and one light atom in the gerade state. Within the BO approach the three-body problem reduces to the calculation of the relative motion of the heavy atoms in the effective potential created by the light atom. For the light atom in the gerade state, this potential is $\epsilon_{+}(R)$ found in the previous subsection. The Schrödinger equation for the wavefunction of the relative motion of the heavy atoms, $\chi_{\nu}(\mathbf{R})$, reads:

$$
\hat{H} \chi(\mathbf{R})=\left[-\left(\hbar^{2} / M\right) \nabla_{\mathbf{R}}^{2}+\epsilon_{+}(R)\right] \chi_{\nu}(\mathbf{R})=\epsilon_{\nu} \chi_{\nu}(\mathbf{R}) .
$$

The trimer states are nothing else than the bound states of heavy atoms in the effective potential $\epsilon_{+}(R)$. Accordingly, they correspond to the discrete part of the spectrum $\epsilon_{\nu}$, where the symbol $\nu$ denotes a set containing angular $(l)$ and radial $(n)$ quantum numbers. For $R \ll a$ the potential $\epsilon_{+}(R)$ is proportional to $-1 / R^{2}$ (see Eq. (42)) and, if this effective attraction overcomes the centrifugal barrier, we arrive at the well known phenomenon of the fall of a particle to the center in an attractive $1 / R^{2}$ potential. Then, for a given orbital angular 
momentum $l$, the radial part of $\chi_{\nu}$ can be written as

$$
\chi_{\nu}(R) \propto R^{-1 / 2} \sin \left(s_{l} \ln R / r_{0}\right), \quad R \ll a,
$$

where

$$
s_{l}=\sqrt{0.16 M / m-(l+1 / 2)^{2}} .
$$

The three-body parameter $r_{0}$ determines the phase of the wavefunction at small distances and, in principle, depends on $l$. The wavefunction (56) has infinitely many nodes, which means that in the zero-range approximation there are infinitely many trimer states. This is one of the properties of three-body systems with resonant interactions discovered by Efimov [53. We see that the fall to the center is possible in many angular momentum channels, provided the mass ratio is sufficiently large. However, for practical purposes and for simplicity, it is sufficient to consider the case where the Efimov effect occurs only for the angular momentum channel with the lowest possible $l$ for a given symmetry. This implies that when the heavy atoms are fermions and one has odd $l$, in order to confine ourselves to $l=1$ we should have the mass ratio in the range $14 \lesssim M / m \lesssim 76$. For bosonic heavy atoms where $l$ is even, we set $l=0$ and consider $M / m \lesssim 39$ to avoid the Efimov effect for $l \geq 2$. In both cases we need a single three-body parameter $r_{0}$.

The formation of Efimov trimers in ultracold dimer-dimer collisions is energetically allowed only if $\epsilon_{\nu}<-2\left|\epsilon_{0}\right|$. This means that the trimers that we are interested in are relatively well bound and their size is smaller than $a$. Therefore, the process of the trimer formation is exponentially reduced for large mass ratios as the heavy atoms have to tunnel under the repulsive barrier $U_{\text {eff }}(\mathrm{R})$. Moreover, this process requires all of the four atoms to approach each other at distances smaller than $a$, and its rate decreases with the trimer size because it is more difficult for two identical light fermions to be in a smaller volume.

From Eq. (56) one sees that the behavior of the three-body system does not change if $r_{0}$ is multiplied by

$$
\lambda_{l}=\exp \left(\pi / s_{l}\right)
$$

On the other hand, the dimensional analysis shows that the quantity $\epsilon_{\nu} / \epsilon_{0}$ depends only on the ratio $a / r_{0}$. This means that except for a straightforward scaling with $a$, properties of the three-body system do not change when $a$ is multiplied or divided by $\lambda_{l}$. This discrete scaling symmetry of a three-body system, which shows itself in the log-periodic dependence of three-body observables, has yet to be observed experimentally. In the case of three identical bosons, where the BO approach does not work and it is necessary to solve the three-body problem exactly [53, the observation of the consequences of the discrete scaling requires to change $a$ by a factor of $\lambda \approx 22.7$, which is technically very difficult in ongoing experiments with cold atoms. In this respect three-body systems with a very large mass difference can be more favorable because of smaller values of $\lambda$. For example, in order to see one period of the log-periodic dependence in a Cs-Cs-Li three-body system $a$ has to be changed only by a factor of $\lambda \approx 5$. 
At this point it is worth emphasizing that three-body effects can be observed in a gas of light-heavy dimers, where the interdimer repulsion originating from the exchange of the light fermions strongly reduces the decay rate associated with relaxation of the dimers into deep bound states. The trimer formation in dimer-dimer collisions is very sensitive to the positions and sizes of Efimov states, and the measurement of the formation rate can be used to demonstrate the discrete scaling symmetry of a three-body system. Indeed, this rate should have the log-periodic dependence on $a$ and is detectable by measuring the lifetime of the gas of dimers.

Besides the Efimov trimers, one can have of one light and two heavy atoms may form "universal" trimer states well described in the zero-range approximation without introducing the three-body parameter 69. In particular, they exist for the orbital angular momentum $l=1$ and mass ratios below the critical

value, where the Efimov effect is absent and short-range physics drops out of consideration. One of such states emerges at $M / m \approx 8$ and crosses the trimer formation threshold $\left(\epsilon_{t r}=-2\left|\epsilon_{0}\right|\right)$ at $M / m \approx 12.7$. The existence of this state is already seen in the $\mathrm{BO}$ picture. It appears as a bound state of fermionic heavy atoms in the potential $\epsilon_{+}(R)$ for $l=1$. The other state exists at $M / m$ even closer to the critical mass ratio and never becomes sufficiently deeply bound to be formed in cold dimer-dimer collisions. The universal trimer states also exist for $l>1$ and $M / m>13.6$ [69. However, the trimer formation in dimer-dimer collisions at such mass ratios is dominated by the contribution of Efimov trimers with smaller $l$. Therefore, below we focus on the formation of Efimov trimers.

The calculation of the intrinsic lifetime of a trimer requires a detailed knowledge of short-range physics and is a tedious task. Estimates of the imaginary part of the trimer energy, $\tau^{-1}$, from the experimental data on $\mathrm{Cs}_{3}$ trimers [70] show that it is approximately by a factor of 4 smaller than the real part $\epsilon_{\nu}$ (in this case $\eta_{*} \approx 0.06$ ). From a general point of view, we do not expect that the trimers with a binding energy $\epsilon_{\nu}<-2\left|\epsilon_{0}\right|$ are very long-lived. However, one can have relatively narrow resonances, and we demonstrate calculations for various values of the elasticity parameter $\eta_{*}[67$.

\subsection{Collisional relaxation of molecules of heavy and light fermions and formation of trimers}

Let us now discuss inelastic processes in dimer-dimer collisions and start with relaxation of the dimers into deeply bound states. The typical size of a deeply bound state is of the order of the characteristic radius of the corresponding interatomic potential. We first consider the relaxation channel that requires one light and two heavy atoms to approach each other at distances $\sim R_{e} \ll a$. Unlike the trimer formation, this decay mechanism is a purely three-body process. The other light atom is just a spectator. A qualitative scenario of this process is the following. With the tunneling probability which is exponentially suppressed for large $M / m$, two dimers approach each other at distances $R \sim a$. Then the heavy atoms are accelerated towards each other in the potential $\epsilon_{+}(R)$, and the light atom in the gerade state is always closely bound to the heavy ones as is 
seen from the shape of the function $\psi^{+}$. The relaxation transition occurs when the heavy atoms (and the gerade light fermion) are at interatomic separations $\sim R_{e}$. The relaxation rate constant certainly satisfies Eq. (48), but we should also find out how to take into account the relaxation process in the description of the trimer states and their formation. The most convenient way to do so is to consider the three-body parameter $r_{0}$ as a complex quantity and introduce the so-called elasticity parameter $\eta_{*}=-s_{l} \operatorname{Arg}\left(r_{0}\right)$ [71. As follows from the asymptotic expression for the wavefunction (56), a negative argument of $r_{0}$ ensures that the incoming flux of heavy atoms is not smaller than the outgoing one:

$$
\Phi_{\text {out }} / \Phi_{\text {in }}=\exp \left[4 s_{l} \operatorname{Arg}\left(r_{0}\right)\right]=\exp \left[-4 \eta_{*}\right] \leq 1
$$

This mimics the loss of atoms at small distances due to the relaxation into deeply bound states. In the analysis of Efimov states, the imaginary part of $r_{0}$ leads to the appearance of an imaginary part of $\epsilon_{\nu}$. This means that any Efimov state has a finite lifetime $\tau$ due to the relaxation. For small $\left|\operatorname{Arg}\left(r_{0}\right)\right|$ and for trimer states that are localized at distances smaller than $a$, we get $\tau^{-1} /\left|\epsilon_{\nu}\right|=4\left|\operatorname{Arg}\left(r_{0}\right)\right|=4 \eta_{*} / s_{l}$. Strictly speaking this fact indicates that it is not possible to separate the relaxation process from the trimer formation because the trimers that are formed in dimer-dimer collisions will eventually decay due to the relaxation. Nevertheless, both the modulus and the argument of the three-body parameter can be determined by measuring the lifetime of a gas of dimers, leading to a number of quantitative predictions concerning the structure of Efimov states in the three-body subsystem of one light and two heavy atoms.

Another relaxation channel is the one in which two light atoms approach a heavy atom at distances $\sim R_{e} \ll a$. This channel is, however, suppressed due to the Fermi statistics for the light atoms, which strongly reduces the probability of having them in a small volume. As a result, for realistic parameters this relaxation mechanism is much weaker than the one in the system of one light and two heavy atoms [67.

The study of the formation of trimer states in molecule-molecule collisions requires us to go beyond the conventional Born-Oppenheimer approximation, since this approximation breaks down for the ungerade light atom at separations between the heavy atoms $R<a$. Within the recently developed "hybrid Born-Oppenheimer" approach 67] one applies the Born-Oppenheimer method to the gerade light fermion which is characterized by the wavefunction $\psi_{\mathbf{R}}^{+}(\mathbf{r})$ and energy $\epsilon_{+}(R)$ adiabatically ajusting themselves to the motion of the heavy atoms. The gerade light atom is then integrated out by introducing the potential $\epsilon_{+}(R)$ for the heavy atoms. Once this is done the original 4-body problem is reduced to a 3-body problem described by the Schrödinger equation equation

$$
\left[\hat{H}-\left(\hbar^{2} / 2 \mu_{3}\right) \nabla_{\mathbf{r}}^{2}-E\right] \Psi(\mathbf{R}, \mathbf{r})=0,
$$

where $\hat{H}$ is given by Eq. (55), $\mu_{3}=2 m M /(2 M+m), E=-2\left|\epsilon_{0}\right|+\epsilon$ is the total energy of the four-body system in the center of mass reference frame, and $\epsilon$ is the dimer-dimer collision energy. This problem is then treated exactly in the 
Bethe-Peierls approach, and the interaction of the light atom with the heavy atoms is included in the form of the Bethe-Peierls boundary condition (8) for $\Psi$ at vanishing light-heavy separations $|\mathbf{r} \pm \mathbf{R} / 2|$. The ungerade symmetry for this atom is taken into account by the condition

$$
\Psi(\mathbf{R}, \mathbf{r})=-\Psi(\mathbf{R},-\mathbf{r}) .
$$

As the heavy atoms are identical fermions, we have $\Psi(\mathbf{R}, \mathbf{r})=-\Psi(-\mathbf{R}, \mathbf{r})$. Combined with Eq. (61), this leads to the condition $\Psi(\mathbf{R}, \mathbf{r})=\Psi(-\mathbf{R},-\mathbf{r})$. Therefore, $\Psi(\mathbf{R}, \mathbf{r})$ describes atom-dimer scattering with even angular momenta, and for ultracold collisions we have to solve an $s$-wave atom-dimer scattering problem.

In order to solve Eq. (60) we follow the method of Ref. [54 and introduce an auxiliary function $f(\mathbf{R})$ and write down the wavefunction $\Psi(\mathbf{R}, \mathbf{r})$ in the form:

$$
\Psi(\mathbf{R}, \mathbf{r})=\sum_{\nu} \int_{\mathbf{R}^{\prime}} \chi_{\nu}(\mathbf{R}) \chi_{\nu}^{*}\left(\mathbf{R}^{\prime}\right) K_{\kappa_{\nu}}\left(2 \mathbf{r}, \mathbf{R}^{\prime}\right) f\left(\mathbf{R}^{\prime}\right),
$$

where

$$
K_{\kappa_{\nu}}\left(2 \mathbf{r}, \mathbf{R}^{\prime}\right)=\frac{e^{-\kappa_{\nu}\left|\mathbf{r}-\mathbf{R}^{\prime} / 2\right|}}{4 \pi\left|\mathbf{r}-\mathbf{R}^{\prime} / 2\right|}-\frac{e^{-\kappa_{\nu}\left|\mathbf{r}+\mathbf{R}^{\prime} / 2\right|}}{4 \pi\left|\mathbf{r}+\mathbf{R}^{\prime} / 2\right|}
$$

and

$$
\kappa_{\nu}= \begin{cases}\sqrt{2 \mu_{3}\left(\epsilon_{\nu}-E\right)} / \hbar, & \epsilon_{\nu}>E \\ -i \sqrt{2 \mu_{3}\left(E-\epsilon_{\nu}\right)} / \hbar, & \epsilon_{\nu}<E .\end{cases}
$$

For $\epsilon_{\nu}<E$, the trimer can be formed in the state $\nu$. In this case $\kappa_{\nu}$ is imaginary and the function (63) describes an outgoing wave of the light atom moving away from the trimer. The choice of the sign in Eq. (64) ensures that there is no incoming flux in the atom-trimer channel.

Using the Bethe-Peierls boundary condition (8) for the wavefunction (62) at $|\mathbf{r} \pm \mathbf{R} / 2| \rightarrow 0$ we one obtain an integral equation for the function $f(\mathbf{R})$ :

$$
\left\{\hat{L}-\hat{L}^{\prime}+\sin ^{2} \theta \frac{\sqrt{2 \mu\left(\epsilon_{0}-E\right)} / \hbar-1 / a}{4 \pi}\right\} f(\mathbf{R})=0,
$$

where $\mu=m M /(m+M), \theta=\arctan \sqrt{1+2 M / m}$, and

$$
\begin{gathered}
\hat{L}^{\prime} f(\mathbf{R})=\int_{\mathbf{R}^{\prime}} \sum_{\nu}\left[\chi_{\nu}(\mathbf{R}) \chi_{\nu}^{*}\left(\mathbf{R}^{\prime}\right) K_{\kappa_{\nu}}\left(\mathbf{R}, \mathbf{R}^{\prime}\right)-\chi_{\nu}^{0}(\mathbf{R}) \chi_{\nu}^{0 *}\left(\mathbf{R}^{\prime}\right) K_{\kappa_{\nu}^{0}}\left(\mathbf{R}, \mathbf{R}^{\prime}\right)\right] f\left(\mathbf{R}^{\prime}\right), \\
\hat{L} f(\mathbf{R})=P \int_{\mathbf{R}^{\prime}}\left\{G\left(\left|\mathbf{R}-\mathbf{R}^{\prime}\right|\right)\left[f(\mathbf{R})-f\left(\mathbf{R}^{\prime}\right)\right] \pm G\left(\sqrt{R^{2}+R^{\prime 2}-2 \mathbf{R} \cdot \mathbf{R}^{\prime} \cos 2 \theta}\right) f\left(\mathbf{R}^{\prime}\right)\right\} \\
G(X)=\frac{\sin 2 \theta M\left(\epsilon_{0}-E\right) K_{2}\left(\sqrt{M\left(\epsilon_{0}-E\right)} X / \hbar \sin \theta\right)}{8 \hbar^{2} \pi^{3} X^{2}}
\end{gathered}
$$

with $K_{2}(z)$ being the exponentially decaying Bessel (Macdonald) function. A detailed derivation is given in Ref. [67] and is omitted here. The operators $\hat{L}$ and 
$\hat{L}^{\prime}$ conserve angular momentum and, expanding the function $f(\mathbf{R})$ in spherical harmonics, we arrive at a set of uncoupled 1D integral equations for each of the radial functions $f_{l}(R)$. Below we present the results for $s$-wave dimer-dimer scattering.

At large distances $(R \gg a)$ the reduced wavefunction $\Psi(\mathbf{R}, \mathbf{r})$ takes the form:

$$
\Psi(\mathbf{R}, \mathbf{r}) \approx \Psi(\mathbf{R}) \psi_{\mathbf{R}}^{-}(\mathbf{r}),
$$

and one can show that

$$
\Psi(\mathbf{R}) \propto f(\mathbf{R}) ; \quad R \gg a .
$$

Therefore, $f(\mathbf{R})$ can serve as the wavefunction for the dimer-dimer motion at large distances. In particular, it contains the dimer-dimer scattering phase shift.

The dimer-dimer $s$-wave scattering amplitude $a_{d d}$ is determined from the asymptotic behavior of the solution of Eq. (65) at large distances for $E=2 \epsilon_{0}$, which should be matched with

$$
f_{0}(R) \propto\left(1 / R-1 / a_{d d}\right)
$$

at $R \gg a \ln \sqrt{M / m}$. In Fig. 11 we compare the resulting $a_{d d} / a$ with that following from Eq. (51). The results agree quite well even for moderate values of $M / m$. These results also agree with the calculations based on the exact fourbody equation for $M / m<13.6$ [65] and displayed in Fig. 8, and with the Monte Carlo results for $M / m<20$ [72].

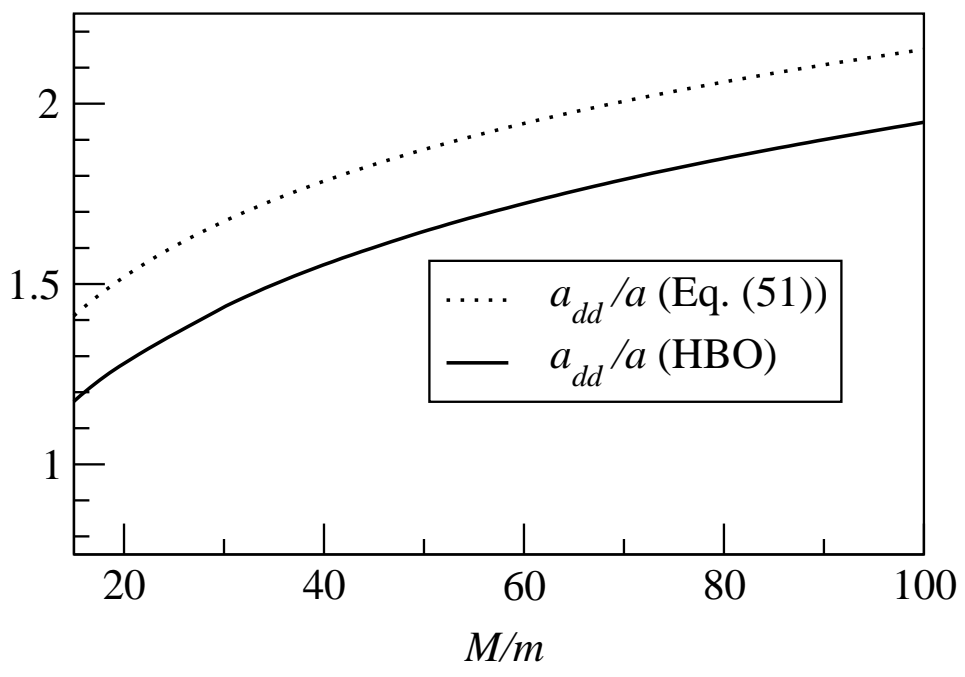

Figure 11: The dimer-dimer $s$-wave scattering length $a_{d d} / a$. The solid curve shows the results obtained in the hybrid Born-Oppenheimer approximation, and the dotted line the results of Eq. (51).

It is straightforward to extend this theory to account for inelastic processes of the trimer formation and the relaxation of dimers into deeply bound states. 
Let us first assume that the rate of the relaxation into deep molecular states is negligible and neglect this process. Then the three-body parameter is real, and the trimer formation rate is determined by the imaginary part of the $s$-wave scattering length. The rate constant is given by 25 .

$$
\alpha=-\frac{16 \hbar \pi}{M} \operatorname{Im}\left(a_{d d}\right) .
$$

Alternatively, if it is necessary to know the rate of the trimer formation in the state $\nu$, one can substitute the solution of Eq. (65) into Eq. (62) and calculate the flux of light atoms at $\mathbf{r} \rightarrow \infty$. The summation over $\nu$ gives the same result as Eq. (72). We find that the contribution of the highest "dangerous" trimer state is by far dominant and $\alpha$ is very sensitive to its position.

We now include the relaxation of the dimers into deeply bound states. As we have mentioned above, the light-heavy-heavy relaxation process can be taken into account by adding an imaginary part to the three-body parameter. The total inelastic decay rate is then still given by Eq. (72). However, strictly speaking we can no longer distinguish between the formation of the trimer in a particular state and the collisional relaxation since the trimers ultimately decay due to the relaxation process. In this sense the only decay channel is the relaxation. However, for a sufficiently long lifetime of a trimer, i.e. if the trimer states are narrow resonances, we can still observe a pronounced dependence of the total inelastic decay rate on the position of the highest "dangerous" trimer state (see below).

Fig. 12 shows the results for the inelastic collisional rate in the case of bosonic molecules with the mass ratio $M / m=28.5$ characteristic of ${ }^{171} \mathrm{Yb}-{ }^{6} \mathrm{Li}$ dimers. The solid line corresponds to the case of a real three-body parameter. It is convenient to introduce a related quantity, $a_{0}$, defined as the value of $a$ at which the energy, $\epsilon_{\nu}$, of a trimer state exactly equals $E=2 \epsilon_{0}$. This new "dangerous" trimer state becomes more deeply bound for $a>a_{0}$ and the rate constant rapidly increases. It is proportional to the density of states in the outgoing atom-trimer channel. The corresponding orbital angular momentum is equal to 1 and the threshold law reads (see also inset in Fig. 12):

$$
\alpha \propto \text { const }+\left(E-\epsilon_{\nu}\right)^{3 / 2} \propto \text { const }+\left(a-a_{0}\right)^{3 / 2} .
$$

The constant term in Eq. (73) describes the contribution of more deeply bound states, which is typically very small. In fact, as trimer states become more compact, both light atoms should approach the heavy atoms and each other to small distances where the trimer formation takes place. Since they are identical fermions, there is a strong suppression of the trimer formation to these deeply bound states.

The dependence of $\alpha$ on $a / a_{0}$ is periodic on the logarithmic scale, the multiplicative factor being equal to $\lambda_{1} \approx 7.3$. The dashed, dotted, and dash-dotted curves are obtained for $\eta_{*}=0.1,0.5$, and 1 , respectively. The corresponding values of the ratio $\Phi_{\text {out }} / \Phi_{\text {in }}$ are $0.67,0.14$, and 0.02 . The horizontal line represents the limiting case of $\eta_{*}=\infty$ or $\Phi_{\text {out }}=0$. This case is universal in the sense 


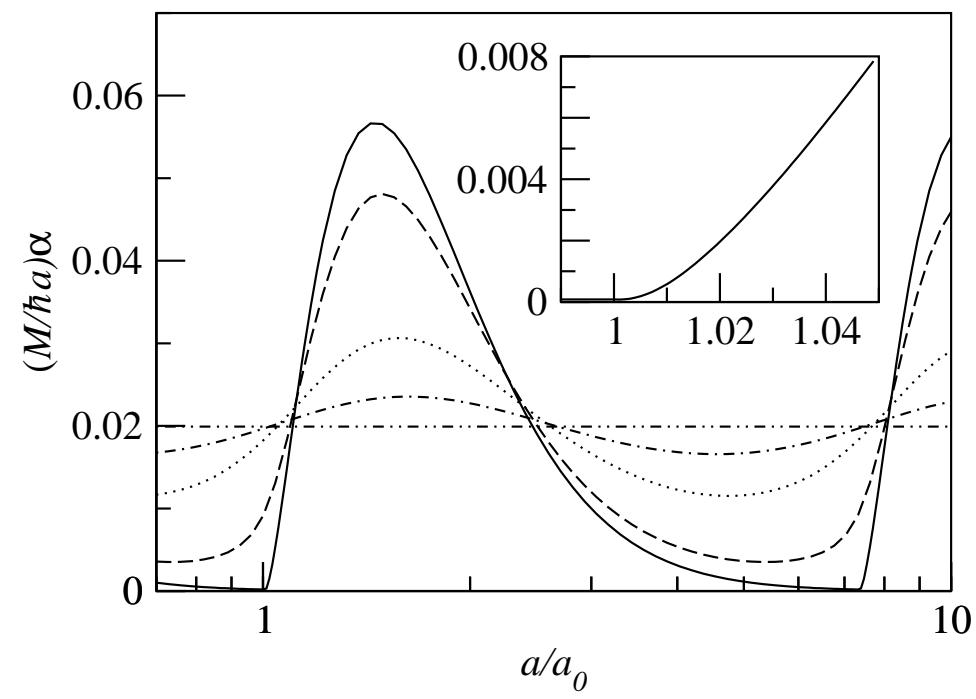

Figure 12: The inelastic rate constant for bosonic dimers with $M / m=28.5$ as a function of the atom-atom scattering length $a$. The solid line corresponds to the case of a real three-body parameter. The results plotted in dashed, dotted, dash-dotted, and dash-dot-dot lines are obtained by taking into account the light-heavy-heavy relaxation processes. The values of the elasticity parameter $\eta_{*}=0.1,0.5,1$, and $\infty$, respectively (see text). The quantity $a_{0}$ is the value of $a$ at which the energy of a trimer state equals $E=2 \epsilon_{0}$ and a new inelastic channel opens. The inset shows the region $a \approx a_{0}$ in greater detail in order to see the threshold behavior (73).

that physical observables depend only on the masses and the atomic scattering length.

For a very weak light-heavy-heavy relaxation, the dimer-dimer inelastic collision can be viewed as the formation of a trimer (with the rate constant $\alpha$ ) followed by its slow decay due to the relaxation. In this case one can think of detecting the trimers spectroscopically. We note, however, that even for the conditions corresponding to the dashed curve in Fig. 12, i.e. for $\eta_{*}$ as small as 0.1 , the decay rate of the trimer $\tau^{-1} \approx 0.25\left|\epsilon_{\nu}\right| / \hbar$ is rather fast, which will likely make its direct detection difficult. For larger $\eta_{*}$ it is impossible to separate the formation of trimers from their intrinsic relaxational decay, and $\alpha$ is practically the relaxation rate constant. Remarkably, it remains quite sensitive to the positions of trimer states (in this case resonances) for values of $\eta_{*}$ up to 0.5 and even larger. This suggests that measuring the lifetime of a gas of dimers as a function of $a$ may provide important information on three-body observables. Moreover, for small $\eta_{*}$ it may be possible to crate a stable molecular gas in sufficiently broad regions of $a$, where "dangerous" trimer states are far from the trimer formation threshold. 
The inelastic rate $\alpha$ for other mass ratios in the range $20<M / m<76$ has also been found 67. Its dependence on the scattering length has the same form as depicted in Fig. 12, The maximum of the rate constant is well fitted by the formula $\alpha_{\max }=5.8(\hbar a / M) \exp (-0.87 \sqrt{M / m})$, and the position of the horizontal line $\left(\eta_{*}=\infty\right)$ by $\alpha_{\infty}=1.6(\hbar a / M) \exp (-0.82 \sqrt{M / m})$. The multiplicative factor in the log-periodic dependence is given by Eqs. (58) and (57) with $l=1$.

The same method was employed to estimate the formation of the universal trimer state with the orbital angular momentum $l=1$ at mass ratios $M / m>$ 12.7 but below the critical value for the onset of the Efimov effect [67. The rate constant increases with $M / m$ and reaches $\alpha=0.2(\hbar a / M)$ close to the critical mass ratio. This corresponds to the imaginary part of the scattering length $\operatorname{Im} a_{d d} \approx 4 \times 10^{-3} a$, which is by a factor of 300 smaller than the real part of $a_{d d}$ obtained from four-body calculations [65]. Thus, the formation of this state does not change the elastic scattering amplitude $a_{d d}$ shown in Fig. 8.

We can now estimate the collisional rates for ${ }^{171} \mathrm{Yb}^{6}{ }^{6} \mathrm{Li}$ dimers. On the basis of the results in Fig. 12 we find that for $a=20 \mathrm{~nm}$ the upper bound of the inelastic rate constant is $\alpha_{\max } \approx 4 \times 10^{-13} \mathrm{~cm}^{3} / \mathrm{s}$. The elastic rate constant for a thermal gas with $a \sim 20 \mathrm{~nm}$ and $T \sim 100 \mathrm{nK}$ equals

$$
\alpha_{e l} \approx 8 \pi\left|a_{d d}\right|^{2} \sqrt{2 T / M} \sim 4 \times 10^{-11} \mathrm{~cm}^{3} / \mathrm{s} .
$$

Here we used the calculated $s$-wave dimer-dimer scattering length $a_{d d} \approx 1.4 a$. We see that $\alpha_{e l}$ is much larger than $\alpha$ and this inequality becomes even more pronounced for larger $a$ due to the scaling relations $\alpha_{e l} \propto a^{2}$ and $\alpha_{\max } \propto$ $a$. Thus, the gas of molecules of heavy and light fermions is well suited for evaporative cooling towards their Bose-Einstein condensation.

\section{Crystalline molecular phase}

\subsection{Born-Oppenheimer potential in a many-body system of molecules of heavy and light fermions}

Strong long-distance repulsive interaction between weakly bound molecules of light and heavy fermionic atoms has important consequence not only for the relaxation process, but also for macroscopic properties of the molecular system. In contrast to two-component Fermi gases of atoms in different internal states, heteronuclear Fermi-Fermi mixtures can form a molecular crystalline phase even when the mean interparticle separation greatly exceeds the size of the molecule:

$$
\bar{R} \gg a \text {. }
$$

Let us consider a mixture of heavy and light fermionic atoms with equal concentrations and a large positive scattering length for the interaction between them, satisfying the inequality $a \gg R_{e}$. At zero temperature all atoms will be converted into weakly bound molecules and under the condition (75) the molecular size $(\sim a)$ will be much smaller than the mean intermolecular 
separation. Then, using the Born-Oppenheimer approximation and integrating out the motion of light atoms we are left with a system of identical (composite) bosons which is described by the Hamiltonian:

$$
\hat{H}=-\frac{\hbar^{2}}{2 M} \sum_{i} \Delta_{\mathbf{R}_{i}}+\frac{1}{2} \sum_{i \neq j} U_{\text {eff }}\left(R_{i j}\right),
$$

where indices $i$ and $j$ label the bosons, their coordinates are denoted by $\mathbf{R}_{i}$ and $\mathbf{R}_{j}$, and $R_{i j}=\left|\mathbf{R}_{i}-\mathbf{R}_{j}\right|$ is the separation between the $i$-th and $j$-th bosons. Assuming that the motion of light fermions is three-dimensional, the effective repulsive potential $U_{\text {eff }}$ is given by Eq. (44) and is independent of the mass of the heavy atom $M$. Therefore, at a large mass ratio $M / m$ it dominates over the kinetic energy which is inversly proportional to $M$, which may lead to the formation of a crystalline phase.

We will discuss the case where the motion of heavy atoms is confined to two dimensions, while the motion of light atoms can be either $2 \mathrm{D}$ or $3 \mathrm{D}$. It will be shown that the Hamiltonian (76) with $U_{\text {eff }}$ (44), supports the first order quantum gas-crystal transition at $T=0$ [68. This phase transition resembles the one for the flux lattice melting in superconductors, where the flux lines are mapped onto a system of bosons interacting via a 2D Yukawa potential [73. In this case the Monte Carlo studies [74, 75] identified the first order liquidcrystal transition at zero and finite temperatures. Aside from the difference in the interaction potentials, a distinguished feature of our system is related to its stability. The molecules can undergo collisional relaxation into deeply bound states, or form weakly bound trimers. Another subtle question is how dilute the system should be to enable the use of the binary approximation for the molecule-molecule interaction, leading to Eqs. (76) and (44).

Let us first consider the system of $N$ molecules and derive the Born-Oppenheimer interaction potential for this system. Omitting the interaction between light (identical) fermions, it is sufficient to find $N$ lowest single-particle eigenstates, and the sum of their energies will give the interaction potential for the molecules. For the interaction between light and heavy atoms we use the Bethe-Peierls approach, and the wavefunction of a single light atom then reads:

$$
\Psi(\{\mathbf{R}\}, \mathbf{r})=\sum_{i=1}^{N} C_{i} G_{\kappa}\left(\mathbf{r}-\mathbf{R}_{i}\right),
$$

where $\mathbf{r}$ is its coordinate. The Green function $G_{\kappa}$ satisfies the equation $\left(-\nabla_{\mathbf{r}}^{2}+\right.$ $\left.\kappa^{2}\right) G_{\kappa}(\mathbf{r})=\delta(\mathbf{r})$. The energy of the state (77) equals $\epsilon=-\hbar^{2} \kappa^{2} / 2 m$, and here we only search for negative single-particle energies. The dependence of the coefficients $C_{i}$ and $\kappa$ on $\{\mathbf{R}\}$ is obtained using the Bethe-Peierls boundary condition:

$$
\Psi(\{\mathbf{R}\}, \mathbf{r}) \propto G_{\kappa_{0}}\left(\mathbf{r}-\mathbf{R}_{i}\right) ; \quad \mathbf{r} \rightarrow \mathbf{R}_{i} .
$$

Up to a normalization constant, $G_{\kappa_{0}}$ is the wavefunction of a bound state of a single molecule with energy $\epsilon_{0}=-\hbar^{2} \kappa_{0}^{2} / 2 m$ and molecular size $\kappa_{0}^{-1}$. The 
Bethe-Peierls boundary condition (78) written through the Green function $G_{\kappa_{0}}$ can be used for both 2D and 3D motion of light atoms. In the latter case one has $\kappa_{0}=a^{-1}$, the Green function is $G_{\kappa_{0}}\left(\mathbf{r}-\mathbf{R}_{i}\right) \propto\left(\left|\mathbf{r}-\mathbf{R}_{i}\right|^{-1}-a^{-1}\right)$ for $\left|\mathbf{r}-\mathbf{R}_{i}\right| \rightarrow 0$, and Eq. (78) takes the form (8).

From Eqs. (77) and (78) we get a set of $N$ equations: $\sum_{j} A_{i j} C_{j}=0$, where $A_{i j}=\lambda(\kappa) \delta_{i j}+G_{\kappa}\left(R_{i j}\right)\left(1-\delta_{i j}\right), R_{i j}=\left|\mathbf{R}_{i}-\mathbf{R}_{j}\right|$, and $\lambda(\kappa)=\lim _{r \rightarrow 0}\left[G_{\kappa}(r)-\right.$ $G_{\kappa_{0}}(r)$ ]. The single-particle energy levels are determined by the equation

$$
\operatorname{det}\left[A_{i j}(\kappa,\{\mathbf{R}\})\right]=0 .
$$

For $R_{i j} \rightarrow \infty$, Eq. (79) gives an $\mathrm{N}$-fold degenerate ground state with $\kappa=\kappa_{0}$. At finite large $R_{i j}$, the levels split into a narrow band. Given a small parameter

$$
\xi=G_{\kappa_{0}}(\tilde{R}) / \kappa_{0}\left|\lambda_{\kappa}^{\prime}\left(\kappa_{0}\right)\right| \ll 1,
$$

where $\tilde{R}$ is a characteristic distance at which heavy atoms can approach each other, the bandwidth is $\Delta \epsilon \approx 4\left|\epsilon_{0}\right| \xi \ll\left|\epsilon_{0}\right|$. It is important for the adiabatic approximation that all lowest $N$ eigenstates have negative energies and are separated from the continuum by a gap $\sim\left|\epsilon_{0}\right|$.

We now calculate the single-particle energies up to second order in $\xi$. To this order we write $\kappa(\lambda) \approx \kappa_{0}+\kappa_{\lambda}^{\prime} \lambda+\kappa_{\lambda \lambda}^{\prime \prime} \lambda^{2} / 2$ and turn from $A_{i j}(\kappa)$ to $A_{i j}(\lambda)$ :

$$
A_{i j}=\lambda \delta_{i j}+\left[G_{\kappa_{0}}\left(R_{i j}\right)+\kappa_{\lambda}^{\prime} \lambda \partial G_{\kappa_{0}}\left(R_{i j}\right) / \partial \kappa\right]\left(1-\delta_{i j}\right),
$$

where all derivatives are taken at $\lambda=0$. Using $A_{i j}$ (81) in Eq. (79) gives a polynomial of degree $N$ in $\lambda$. Its roots $\lambda_{i}$ give the light-atom energy spectrum $\epsilon_{i}=-\hbar^{2} \kappa^{2}\left(\lambda_{i}\right) / 2 m$. The total energy, $E=\sum_{i=1}^{N} \epsilon_{i}$, is then given by

$$
E=-\left(\hbar^{2} / 2 m\right)\left[N \kappa_{0}^{2}+2 \kappa_{0} \kappa_{\lambda}^{\prime} \sum_{i=1}^{N} \lambda_{i}+\left(\kappa \kappa_{\lambda}^{\prime}\right)_{\lambda}^{\prime} \sum_{i=1}^{N} \lambda_{i}^{2}\right] .
$$

Keeping only the terms up to second order in $\xi$ and using basic properties of determinants and polynomial roots we find that the first order terms vanish, and the energy reads $E=N \epsilon_{0}+(1 / 2) \sum_{i \neq j} U\left(R_{i j}\right)$, where

$$
U(R)=-\frac{\hbar^{2}}{m}\left[\kappa_{0}\left(\kappa_{\lambda}^{\prime}\right)^{2} \frac{\partial G_{\kappa_{0}}^{2}(R)}{\partial \kappa}+\left(\kappa \kappa_{\lambda}^{\prime}\right)_{\lambda}^{\prime} G_{\kappa_{0}}^{2}(R)\right] .
$$

Thus, up to second order in $\xi$ the interaction in the system of $\mathrm{N}$ molecules is the sum of binary potentials (83).

If the motion of light atoms is $3 \mathrm{D}$, the Green function is $G_{\kappa}(R)=(1 / 4 \pi R) \exp (-\kappa R)$, and $\lambda(\kappa)=\left(\kappa_{0}-\kappa\right) / 4 \pi$, with the molecular size $\kappa_{0}^{-1}$ equal to the $3 \mathrm{D}$ scattering length $a$. Equation (83) then gives a repulsive potential $U_{\text {ex }}$ (44) which we now denote as $U_{\mathrm{ex}}^{3 D}$ :

$$
U_{\mathrm{ex}}^{3 D}(R)=4\left|\epsilon_{0}\right|\left(1-\left(2 \kappa_{0} R\right)^{-1}\right) \exp \left(-2 \kappa_{0} R\right) / \kappa_{0} R,
$$

and the criterion (80) reads $\left(1 / \kappa_{0} R\right) \exp \left(-\kappa_{0} R\right) \ll 1$. For the $2 \mathrm{D}$ motion of light atoms we have $G_{\kappa}(R)=(1 / 2 \pi) K_{0}(\kappa R)$ and $\lambda(\kappa)=-(1 / 2 \pi) \ln \left(\kappa / \kappa_{0}\right)$, 
where $K_{0}$ is the decaying Bessel function, and $\kappa_{0}^{-1}$ follows from [76]. This leads to a repulsive intermolecular potential:

$$
U_{\mathrm{ex}}^{2 D}(R)=4\left|\epsilon_{0}\right|\left[\kappa_{0} R K_{0}\left(\kappa_{0} R\right) K_{1}\left(\kappa_{0} R\right)-K_{0}^{2}\left(\kappa_{0} R\right)\right],
$$

with the validity criterion $K_{0}\left(\kappa_{0} R\right) \ll 1$. In both cases, which we denote $2 \times 3$ and $2 \times 2$ for brevity, the validity criteria are well satisfied already for $\kappa_{0} R \approx 2$.

\subsection{Gas-crystal quantum transition}

The inequality $\kappa_{0} \bar{R} \gtrsim 2$ may be considered as the condition under which the system is described by the Hamiltonian (76), with $U_{\text {eff }}$ given by Eq. (84) for the 3D motion of light atoms, or by Eq. 855) in the case where this motion is $2 \mathrm{D}$. The state of the system is then determined by two parameters: the mass ratio $M / m$ and the rescaled $2 \mathrm{D}$ density $n \kappa_{0}^{-2}$. At a large $M / m$, the potential repulsion dominates over the kinetic energy, which should lead to the formation of a crystalline ground state. For separations $R_{i j}<\kappa_{0}^{-1}$ the adiabatic approximation breaks down. However, the interaction potential $U(R)$ is strongly repulsive at larger distances. Hence, even for an average separation between heavy atoms, $\bar{R}$, close to $2 / \kappa_{0}$, they approach each other to distances smaller than $\kappa_{0}^{-1}$ with a small tunneling probability $P \propto \exp (-\beta \sqrt{M / m}) \ll 1$, where $\beta \sim 1$. It is then possible to extend $U_{\mathrm{ex}}^{3 D}(R)$ (or $\left.U_{\mathrm{ex}}^{2 D}(R)\right)$ to $R \lesssim \kappa_{0}^{-1}$ in a way providing a proper molecule-molecule scattering phase shift in vacuum and verify that the phase diagram for the many-body system is not sensitive to the choice of this extension 68 .

In Fig. 13 we display the zero-temperature phase diagram obtained by the Diffusion Monte Carlo method [68. Simulations were performed with 30 particles and showed that the solid phase is a $2 \mathrm{D}$ triangular lattice. For the largest density it has been verified that using more particles has little effect on the results.

For both $2 \times 3$ and $2 \times 2$ cases the (Lindemann) ratio $\gamma$ of the rms displacement of molecules to $\bar{R}$ on the transition lines ranges from 0.23 to 0.27 . At low densities $n$ the de Broglie wavelength of molecules is $\Lambda \sim \gamma \bar{R} \gg \kappa_{0}^{-1}$, and $U_{\mathrm{ex}}^{3 D}(R)$ (or $\left.U_{\mathrm{ex}}^{2 D}(R)\right)$ can be approximated by a hard-disk potential with the diameter equal to the $2 \mathrm{D}$ scattering length. Then, using the DMC results for hard-disk bosons 77, we obtain the transition lines shown by solid curves in Fig. 13. At larger $n$, we have $\Lambda<\kappa_{0}^{-1}$ and using the harmonic expansion of $U(R)$ around equlibrium positions in the crystal, we calculate the Lindemann ratio and select $\gamma$ for the best fit to the Monte Carlo data points (dashed curves in Fig. 13).

\subsection{Molecular superlattice in an optical lattice}

The mass ratio above 100, required for the observation of the crystalline order can be achieved in an optical lattice with a small filling factor for heavy atoms. Their effective mass in the lattice, $M_{*}$, can be made very large, and the discussed 


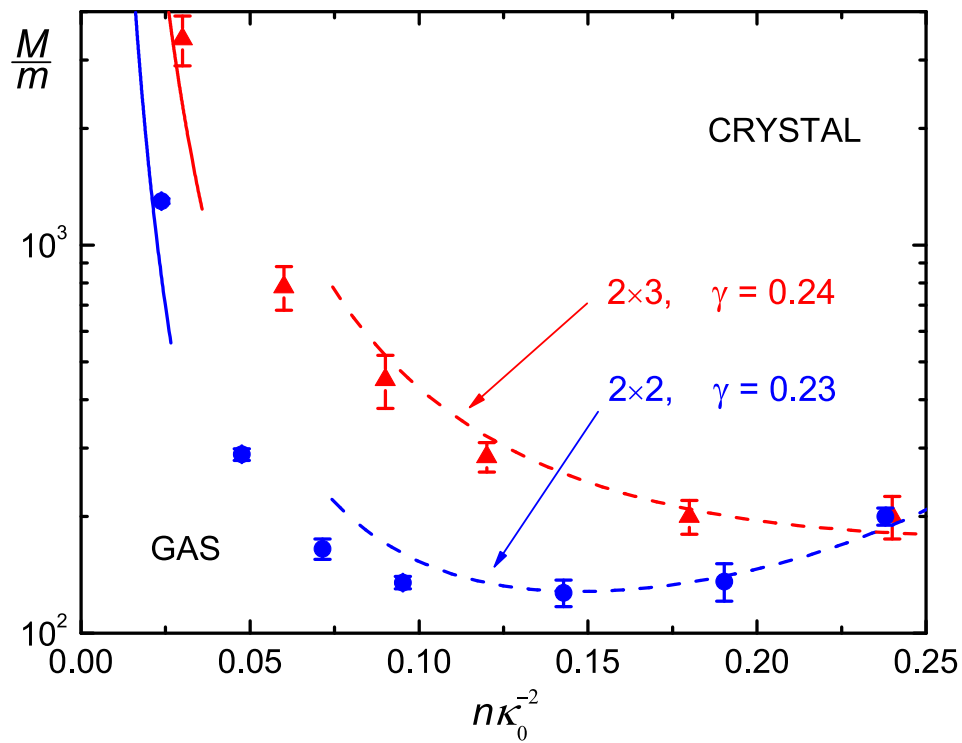

Figure 13: Diffusion Monte Carlo gas-crystal transition lines for 3D (triangles) and 2D (circles) motion of light atoms. Solid curves show the low-density harddisk limit, and dashed curves the results of the harmonic approach (see text).

solid phase should appear as a superlattice. There is no interplay between the superlattice order and the shape of the underlying optical lattice, in contrast to the recently studied solid and supersolid phases in a triangular lattice with the filling factor of order one [78, 79, 80. The superlattice discussed in our review remains compressible and supports two branches of phonons.

The gaseous and solid phases of weakly bound molecules in an optical lattice are metastable. As well as in the gas of such molecules in free space, the main decay channels are the relaxation of molecules into deep bound states and the formation of trimer states by one light and two heavy atoms. The relaxation into deeply bound states turns out to be rather slow, with a relaxation time exceeding $10 \mathrm{~s}$ even at $2 \mathrm{D}$ densities $\sim 10^{9} \mathrm{~cm}^{-2} 68$.

The most interesting is the formation of the trimer states. In an optical lattice the trimers are eigenstates of the Hamiltonian $H_{0}=-\left(\hbar^{2} / 2 M_{*}\right) \sum_{i=1,2} \Delta_{\mathbf{R}_{\mathbf{i}}}+$ $\epsilon_{+}\left(R_{12}\right)$. In a deep lattice it is possible to neglect all higher bands and regard $\mathbf{R}_{\mathbf{i}}$ as discrete lattice coordinates and $\Delta$ as the lattice Laplacian. Then, the fermionic nature of the heavy atoms prohibits them to be on the same lattice site. For a very large mass ratio $M_{*} / m$ the kinetic energy term in $H_{0}$ can be neglected, and the lowest trimer state has energy $\epsilon_{\mathrm{tr}} \approx \epsilon_{+}(L)$, where $L$ is the lattice period. It consists of a pair of heavy atoms localized at neighboring sites and a light atom in the gerade state. Higher trimer states are formed by heavy atoms localized in sites separated by distances $R>L$. This picture breaks down at large $R$, where the spacing between trimer levels is comparable with 
the tunneling energy $\hbar^{2} / M_{*} L^{2}$ and the heavy atoms are delocalized.

In the many-body molecular system the scale of energies in Eq. (76) is much smaller than $\left|\epsilon_{0}\right|$. Thus, the formation of trimers in molecule-molecule "collisions" is energetically allowed only if the trimer binding energy is $\epsilon_{\operatorname{tr}}<2 \epsilon_{0}$. Since the lowest trimer energy in the optical lattice is $\epsilon_{+}(L)$, the trimer formation requires the condition $\epsilon_{+}(L) \lesssim 2 \epsilon_{0}$, which is equivalent to $\kappa_{0}^{-1} \gtrsim 1.6 L$ in the $2 \times 3$ case and $\kappa_{0}^{-1} \gtrsim 1.25 L$ in the $2 \times 2$ case. This means that for a sufficiently small molecular size or large lattice period $L$ the formation of trimers is forbidden.

At a larger molecular size or smaller $L$ the trimer formation is possible. The formation rate has been calculated in Ref. 68 by using the hybrid BornOppenheimer approach, and here we only present the results and give their qualitative explanation. In order to form a bound trimer state two molecules have to tunnel towards each other at distances $R \lesssim \kappa_{0}^{-1}$. This can be viewed as tunneling of particles with mass $M_{*}$ in the repulsive potential $U_{\text {eff }}(R)$. Therefore, the probability of approaching at interheavy separations where the trimer formation occurs, acquires a small factor $\exp \left(-J \sqrt{M_{*} / m}\right)$ with $J \sim 1$, and so does the formation rate. Thus, one can suppress the trimer formation by increasing the ratio $M_{*} / m$. On the other hand, for $M_{*} / m \lesssim 100$ these peculiar bound states can be formed on the time scale $\tau \lesssim 1 \mathrm{~s}$.

Note that the trimer states in an optical lattice, at least the lowest ones, are much more long-lived than in the gas phase. An intrinsic relaxational decay is strongly suppressed as it requires the two heavy atoms of the trimer to approach each other and occupy the same lattice sites. The trimer state can also decay when one of the heavy atoms of the trimer is approached by its own light atom and another light atom at light-heavy separations $\sim R_{e}$. However, this decay channel turns out to be rather slow, with a decay time exceeding tens of seconds even at $2 \mathrm{D}$ densities $n \sim 10^{9} \mathrm{~cm}^{-2}[68$.

\section{Concluding remarks and prospects}

The most distinguishing feature of weakly bound bosonic molecules formed of fermionic atoms is their remarkable collisional stability, despite they are in the highest ro-vibrational state. As we mentioned in the Introduction, the lifetime of such molecules can be of the order of seconds or even tens of seconds at densities $\sim 10^{13} \mathrm{~cm}^{-3}$, depending on the value of the two-body scattering length. This allows for interesting manipulations with these molecules. One of the ideas is related to reaching extremely low temperatures in a gas of fermionic atoms at $a<0$ and achieving the superfluid BCS regime. This regime has not been obtained so far because of difficulties with evaporative cooling of fermionic atoms due to Pauli blocking of their elastic collisions. The route to BCS may be the following. In the first stage, one arranges a deep evaporative cooling of the molecular Bose-condensed gas to temperatures of the order of the chemical potential. Then one converts the molecular BEC into fermionic atoms by

adiabatically changing the scattering length to negative values. This provides 
an additional cooling, and the obtained atomic Fermi gas will have extremely low temperatures $\lesssim 10^{-2} T_{F}$, where $T_{F}$ is the Fermi temperature. The gas can then enter the superfluid BCS regime [81. Moreover, at such temperatures elastic collisions are suppressed by a very strong Pauli blocking and the thermal cloud is in the collisionless regime. This is promising for identifying the BCSpaired state through the observation of collective oscillations or free expansion [82, 83, 19, 16, 84.

It will also be interesting to transfer weakly bound molecules of fermionic atoms to their ground (or less excited) ro-vibrational state. For molecules of bosonic atoms this has been done using two-photon spectroscopy [85, 86, 87] and by magnetically tuned mixing of neighbouring molecular levels, which enables otherwise forbidden radiofrequency transitions 88. Long lifetimes of weakly bound molecules of fermionic atoms at densities $\sim 10^{13} \mathrm{~cm}^{-3}$ may ensure an efficient production of ground state molecules compared to the case of more short-lived molecules of bosonic atoms. One could then extensively study the physics of molecular Bose-Einstein condensation. Moreover, the ground-state heteronuclear molecules have a relatively large permanent dipole moment and can be polarized by an electric field. This may be used to create a gas of dipoles interacting via anisotropic long-range forces, which drastically changes the physics of Bose-Einstein condensation (see, e.g., 89] and references therein).

In the last few years, the observation of the Efimov effect was one of the important goals in the cold atom studies. As we discussed in Section 3, the Efimov trimers in the gas phase are short-lived and rather represent narrow resonances. The Efimov effect then manifests itself in the log-periodic dependence of collision rates on the two-body scattering length. In particular, this is the case for the rate of three-body recombination of atoms [70] and for the rate of trimer formation in molecule-molecule collisions [67. In this sense, the trimer formation in gases of bosonic molecules consisting of heavy and light fermions (such as, for example, LiYb) attracts a great interest as the observation of the Efimov oscillations requires a much smaller change of the two-body scattering length (by a factor of 7 or 5) than in the case of identical bosons.

Of particular interest are the trimer states of two heavy and one light fermion in an optical lattice. For 2D densities $\sim 10^{8} \mathrm{~cm}^{-2}$ the rate of the trimer formation can be of the order of seconds, and these states can be detected optically. As we already mentioned in Section 4, the lattice trimers are long-lived, with a lifetime that can be of the order of tens of seconds. Thus, it is interesting to study to which extent these non-conventional states, in which the heavy atoms are localized in different sites and the light atom is delocalized in between them, can exhibit the Efimov effect.

The creation of a superlattice of molecules in an optical lattice also looks feasible. A promising candidate is the ${ }^{6} \mathrm{Li}^{40} \mathrm{~K}$ mixture as the $\mathrm{Li}$ atom may tunnel freely in a lattice while localizing the heavy $\mathrm{K}$ atoms to reach high mass ratios. A lattice with period $250 \mathrm{~nm}$ and $\mathrm{K}$ effective mass $\mathrm{M}^{*}=20 \mathrm{M}$ provide a tunneling rate $\sim 10^{3} \mathrm{~s}^{-1}$ sufficiently fast to let the crystal form. Near a Feshbach resonance, a value $a=500 \mathrm{~nm}$ gives a binding energy $300 \mathrm{nK}$, and lower temperatures should be reached in the gas. The parameters $n \kappa_{0}^{-2}$ of 
Fig. 13 are then obtained at $2 \mathrm{D}$ densities in the range $10^{7}-10^{8} \mathrm{~cm}^{-2}$ easily reachable in experiments.

\section{Acknowledgements}

The work on this review was financially supported by the IFRAF Institute, by ANR (grants 05-BLAN-0205 and and 06-Nano-014), by the EuroQUAM program of ESF (project Fermix), by Nederlandse Stichtung voor Fundamenteel Onderzoek der Materie (FOM), and by the Russian Foundation for Fundamental Research. LKB is a research unit no. 8552 of CNRS, ENS, and of the University of Pierre et Marie Curie. LPTMS is a mixed research unit no. 8626 of CNRS and University Paris-Sud.

\section{References}

[1] E. M. Lifshitz and L. P. Pitaevskii, "Statistical Physics", (Pergamon Press, Oxford, 1980), Part 2.

[2] "Ultra-cold Fermi gases", Proceedings of the International School of Physics "Enrico Fermi", Course CLXIV, edited by M. Inguscio, W. Ketterle, and C. Salomon, IOS Press, 2007.

[3] M.W. Zwierlein, J.R. Abo-Shaeer, A. Schirotzek, C.H. Schunck, and W. Ketterle, "Vortices and superfluidity in a strongly interacting Fermi gas", Nature 435, 1047 (2005).

[4] M.W. Zwierlein, A. Schirotzek, C.H. Schunck, and W. Ketterle, "Fermionic superfluidity with imbalanced spin populations", Science 311, 492 (2006).

[5] G.B. Partridge, W.H. Li, R.I. Kamar, Y.A. Liao, and R.G. Hulet, "Pairing and phase separation in a polarized Fermi gas", Science 311, 503 (2006).

[6] M.W. Zwierlein, A. Schirotzek, C.H. Schunck, and W. Ketterle, "Direct observation of the superfluid phase transition in ultracold Fermi gases", Nature 442, 54 (2006).

[7] Y. Shin, M.W. Zwierlein, C.H. Schunck, A. Schirotzek, and W. Ketterle, "Observation of phase separation in a strongly interacting imbalanced Fermi gas", Phys. Rev. Lett. 97, 030401 (2006).

[8] G.B. Partridge, W.H. Li, Y.A. Liao, R.G. Hulet, M. Haque, and H.T.C. Stoof, "Deformation of a trapped Fermi gas with unequal spin populations", Phys. Rev. Lett. 97190407 (2006).

[9] C. A. Regal, C. Ticknor, J. L. Bohn, and D. S. Jin, "Creation of ultracold molecules from a Fermi gas of atoms", Nature 424, 47 (2003). 
[10] J. Cubizolles, T. Bourdel, S. J. J. M. F. Kokkelmans, G. V. Shlyapnikov, and C. Salomon, "Production of long-lived ultracold Li-2 molecules from a Fermi gas", Phys. Rev. Lett. 91, 240401 (2003).

[11] S. Jochim, M. Bartenstein, A. Altmeyer, G. Hendl, C. Chin, J. Hecker Denschlag, and R. Grimm, "Pure gas of optically trapped molecules created from fermionic atoms", Phys. Rev. Lett. 91, 240402 (2003).

[12] C. A. Regal, M. Greiner, and D. S. Jin, "Lifetime of molecule-atom mixtures near a Feshbach resonance in 40K", Phys. Rev. Lett. 92, 083201 (2004).

[13] M. Greiner, C. Regal, and D. S. Jin, "Emergence of a molecular BoseEinstein condensate from a Fermi gas", Nature 426, 537 (2003).

[14] C. A. Regal, M. Greiner, and D. S. Jin, "Observation of resonance condensation of fermionic atom pairs", Phys. Rev. Lett. 92, 040403 (2004).

[15] S. Jochim, M. Bartenstein, A. Altmeyer, G. Hendl, S. Riedl, C. Chin, J. H. Denschlag, and R. Grimm, "Bose-Einstein condensation of molecules", Science 302, 2101 (2003); M. Bartenstein, A. Altmeyer, S. Riedl, S. Jochim, C. Chin, J. Hecker Denschlag, and R. Grimm, "Crossover from a molecular Bose-Einstein condensate to a degenerate Fermi gas", Phys. Rev. Lett. 92, 120401 (2004).

[16] M. Bartenstein, A. Altmeyer, S. Riedl, S. Jochim, C. Chin, J. Hecker Denschlag, and R. Grimm, "Collective excitations of a degenerate gas at the BEC-BCS crossover", Phys. Rev. Lett. 92, 203201 (2004).

[17] M. W. Zwierlein, C. A. Stan, C. H. Schunck, S. M. F. Raupach, S. Gupta, Z. Hadzibabic, and W. Ketterle, "Observation of Bose-Einstein condensation of molecules", Phys. Rev. Lett. 91, 250401 (2003).

[18] M. W. Zwierlein, C. A. Stan, C. H. Schunck, S. M. F. Raupach, A. J. Kerman, and W. Ketterle, "Condensation of pairs of fermionic atoms near a Feshbach resonance", Phys. Rev. Lett. 92, 120403 (2004).

[19] T. Bourdel, L. Khaykovich, J. Cubizolles, J. Zhang, F. Chevy, M. Teichmann, L. Tarruell, S. J. J. M. F. Kokkelmans, and C. Salomon, "Experimental study of the BEC-BCS crossover region in lithium 6", Phys. Rev. Lett. 93, 050401 (2004).

[20] G.B. Partridge, K.E. Streker, R.I. Kamar, M.W. Jack, and R. G. Hulet, "Molecular probe of pairing in the BEC-BCS crossover", Phys. Rev. Lett. 95, 020404 (2005).

[21] J. Joseph, B. Clancy, L. Luo, J. Kinast, A. Turlapov, and J.E. Thomas, "Measurement of sound velocity in a Fermi gas near a Feshbach resonance", Phys. Rev. Lett. 98, 170401 (2007). 
[22] M. Taglieber, A.C. Voigt, T. Aoki, T.W. Haensch, and K. Dieckmann, "Quantum degenerate two-species Fermi-Fermi mixture coexisting with a Bose-Einstein condensate", Phys. Rev. Lett. 100, 010401 (2008).

[23] E. Wille, F. M. Spiegelhalder, G. Kerner, D. Naik, A. Trenkwalder, G. Hendl, F. Schreck, R. Grimm, T. G. Tiecke, J. T. Walraven, S. J. Kokkelmans, E. Tiesinga, and P. S. Julienne, "Exploring an ultracold fermi-fermi mixture: Interspecies Feshbach resonances and scattering properties of Li-6 and K-40", Phys. Rev. Lett. 100, 053201 (2008).

[24] G. Breit and E. Wigner, "Capture of Slow Neutrons", Phys. Rev. 49, 519 (1936).

[25] L. D. Landau and E. M. Lifshitz, "Quantum Mechanics", (ButterworthHeinemann, Oxford, 1999).

[26] H. Feshbach, "A unified theory of nuclear reactions II", Ann. Phys. 19, 287 (1962); Theoretical Nuclear Physics, (Wiley, New York, 1992).

[27] U. Fano, "Effects of configuration interaction on intensities and phase shifts", Phys. Rev. 124, 1866 (1961).

[28] A. J. Moerdijk, B. J. Verhaar, and A. Axelsson, "Resonances in ultracold collisions of Li-6, Li-7, and Na-23", Phys. Rev. A 51, 4852 (1995); see also E. Tiesinga, B. J. Verhaar, and H. T. C. Stoof, "Threshold and resonance phenomena in ultracold ground-state collisions", Phys. Rev. A 47, 4114 (1993).

[29] P. O. Fedichev, Yu. Kagan, G. V. Shlyapnikov, and J. T. M. Walraven, "Influence of nearly resonant light on the scattering length in low-temperature atomic gases", Phys. Rev. Lett. 77, 2913 (1996).

[30] J. L. Bohn and P. S. Julienne, "Semianalytic theory of laser-assisted resonant cold collisions", Phys. Rev. A 60, 414 (1999).

[31] M. Theis, G. Thalhammer, K. Winkler, M. Hellwig, G. Ruff, R. Grimm, and J. Hecker Denschlag, "Tuning the scattering length with an optically induced Feshbach resonance", Phys. Rev. Lett. 93, 123001 (2004).

[32] G. Thalhammer, M. Theis, K. Winkler, R. Grimm, and J. Hecker Denschlag, "Inducing an optical Feshbach resonance via stimulated Raman coupling", Phys. Rev. A 71, 033403 (2005).

[33] D. M. Eagles, "Possible pairing without superconductivity at low carrier concentrations in bulk and thin-film superconducting semiconductors", Phys. Rev. 186, 456 (1969).

[34] A. J. Leggett, "Diatomic molecules and Cooper pairs", in Modern Trends in the Theory of Condensed Matter, edited by A. Pekalski and J. Przystawa (Springer, Berlin, 1980). 
[35] P. Nozieres and S. Schmitt-Rink, "Bose condensation in an attractive Fermi gas - From weak to strong coupling superconductivity", J. Low Temp. Phys. 59, 195 (1985).

[36] See for review M. Randeria, "Crossover from BCS theory to Bose-Einstein condensation", in Bose-Einstein Condensation, edited by A. Griffin, D. W. Snoke, and S. Stringari (Cambridge University Press, Cambridge, 1995).

[37] K. Miyake, "Fermi-liquid theory of dilute submonolayer ${ }^{3} \mathrm{He}$ on thin ${ }^{4} \mathrm{He}$ film - Dimer bound state and Cooper pairs", Progr. Theor. Phys. 69, 1794 (1983).

[38] See for review M. Yu. Kagan, "Fermi-gas approach to the problem of superfluidity in 3-dimensional and 2-dimensional solutions of He-3 in He-4", Sov. Physics Uspekhi 37, 69 (1994).

[39] M. Holland, S. J. J. M. F. Kokkelmans, M. L. Chiofalo, and R. Walser, "Resonance superfluidity in a quantum degenerate Fermi gas", Phys. Rev. Lett. 87, 120406 (2001).

[40] E. Timmermans, K. Furuya, P.W. Milonni, A.K. Kerman, "Prospect of creating a composite Fermi-Bose superfluid", Phys. Lett. A 285, 228 (2001).

[41] D.S. Petrov, M.A. Baranov, and G.V. Shlyapnikov, "Superfluid transition in quasi-two-dimensional Fermi gases", Phys. Rev. A 67, 031601 (2003).

[42] G. M. Bruun and C. Pethik, "Effective theory of Feshbach resonances and many-body properties of Fermi gases", Phys. Rev. Lett. 92, 140404 (2004).

[43] G. M. Bruun, "Universality of a two-component Fermi gas with a resonant interaction", Phys. Rev. A 70, 053602 (2004).

[44] S. De Palo, M. L. Chiofalo, M. J. Holland, and S. J. J. M. F. Kokkelmans, "Resonance effects on the crossover of bosonic to fermionic superfluidity", Phys. Lett. A 327, 490 (2004).

[45] E. A. Cornell, "Discussion on Fermi gases", KITP Conference on Quantum Gases, Santa Barbara, May 10-14, 2004.

[46] R. Diener and T. -L. Ho, "The condition for universality at resonance and direct measurement of pair wavefunctions using rf spectroscopy", cond-mat/0405174.

[47] D. S. Petrov, "Three-boson problem near a narrow Feshbach resonance", Phys. Rev. Lett. 93, 143201 (2004).

[48] D. S. Petrov, C. Salomon, and G. V. Shlyapnikov, "Weakly bound dimers of fermionic atoms", Phys. Rev. Lett. 93, 090404 (2004).

[49] D. S. Petrov, C. Salomon, and G. V. Shlyapnikov, "Scattering properties of weakly bound dimers of fermionic atoms", Phys. Rev. A 71, 012708 (2005). 
[50] P. D. Drummond and K. Kheruntsyan, "Coherent molecular bound states of bosons and fermions near a Feshbach resonance", Phys. Rev. A 70, 033609 (2004).

[51] Experimental studies of a narrow resonance with ${ }^{6} \mathrm{Li}_{2}$ molecules have been performed at Rice: K. E. Strecker, G. B. Partridge, and R. G. Hulet, "Conversion of an atomic Fermi gas to a long-lived molecular Bose gas", Phys. Rev. Lett. 91, 080406 (2003).

[52] S. J. J. M. F. Kokkelmans, G. V. Shlyapnikov, and C. Salomon, "Degenerate atom-molecule mixture in a cold Fermi gas", Phys. Rev. A 69, 031602 (2004).

[53] V. N. Efimov, "Energy levels arising from resonant two-body forces in a three-body system", Phys. Lett. 33, 563 (1970); "Weakly-bound states of 3 resonantly-interacting particles", Sov. J. Nucl. Phys. 12, 589 (1971); "Energy levels of three resonantly interacting particles", Nucl. Phys. A 210, 157 (1973).

[54] D. S. Petrov, "Three-body problem in Fermi gases with short-range interparticle interaction", Phys. Rev. A 67, 010703 (2003).

[55] G. V. Skorniakov and K. A. Ter-Martirosian, "Three-body problem for short range forces I. Scattering of low-energy neutrons by deutrons", Sov. Phys. JETP 4, 648 (1957).

[56] G. S. Danilov, "On the 3-body problem with short-range forces", Sov. Phys. JETP 13, 349 (1961).

[57] H. Bethe and R. Peierls, "Quantum Theory of the Diplon", Proc. R. Soc. London, Ser. A 148, 146 (1935).

[58] P. Pieri and G. C. Strinati, "Strong-coupling limit in the evolution from BCS superconductivity to Bose-Einstein condensation", Phys. Rev. B 61, 15370 (2000).

[59] G. E. Astrakharchik, J. Boronat, J. Casulleras, and S. Giorgini, "Equation of state of a Fermi gas in the BEC-BCS crossover: A quantum Monte Carlo study", Phys. Rev. Lett. 93, 200404 (2004).

[60] I.V. Brodsky, M.Y. Kagan, A.V. Klaptsov, R. Combescot, and X. Leyronas, "Exact diagrammatic approach for dimer-dimer scattering and bound states of three and four resonantly interacting particles", Phys. Rev. A 73, 032724 (2006).

[61] J. Levinsen and V. Gurarie, "Properties of strongly paired fermionic condensates", Phys. Rev. A 73, 053607 (2006). 
[62] Including $p$-wave interactions, one can think of the formation of deeply bound states by two identical ( $\uparrow$ or $\downarrow$ ) fermions. So, the collision of two weakly bound molecules can lead to the creation of a deep bound state by two $\uparrow$ (or $\downarrow$ ) fermionic atoms, and two $\downarrow$ (or $\uparrow$ ) atoms become unbound.

[63] J, Fuchs, G.J. Duffy, G. Veeravalli, P. Dyke, M. Bartenstein, C.J. Vale, P. Hannaford, and W.J. Rowlands, "Molecular Bose-Einstein condensation in a versatile low power crossed dipole trap", arXiv:0709.2212.

[64] Y. Inada, M. Horikoshi, S. Nakajima, M. Kuwata-Gonokami, M. Ueda, and T. Mukaiyama, "Critical temperature and condensate fraction of a fermion pair condensate", arXiv:0712.1445.

[65] D.S. Petrov, C. Salomon, and G.V. Shlyapnikov, "Diatomic molecules in ultracold Fermi gases - novel composite bosons", J. Phys. B, 38, S645 (2005).

[66] The Born-Oppenheimer approach for the three-body system of one light and two heavy atoms was discussed in: A. C. Fonseca, E. F. Redish, and P. E. Shanley, "Efimov effect in a solvable model", Nucl. Phys. A 320, 273 (1979).

[67] B. Marcelis, S.J.J.M.F. Kokkelmans, G.V. Shlyapnikov, and D.S. Petrov, "Collisional properties of weakly bound heteronuclear dimers", Phys. Rev. A 77, 032707 (2008).

[68] D. S. Petrov, G. E. Astrakharchik, D. J. Papoular, C. Salomon, and G. V. Shlyapnikov, "Crystalline phase of strongly interacting Fermi mixtures", Phys. Rev. Lett. 99, 130407 (2007).

[69] O.I. Kartavtsev and A.V. Malykh, "Low-energy three-body dynamics in binary quantum gases", J. Phys. B 40, 1429 (2007); "Universal description of the rotational-vibrational spectrum of three particles with zero-range interactions", Pis'ma Zh. Eksp. Teor. Fiz. 86, 713 (2007).

[70] T. Kraemer, M. Mark, P. Waldburger, J.G. Danzl, C. Chin, B. Engeser, A.D. Lange, K. Pilch, A. Jaakkola, H.-C. Nagerl, and R. Grimm, "Evidence for Efimov quantum states in an ultracold gas of caesium atoms", Nature (London) 440, 315 (2006).

[71] E. Braaten and H.-W. Hammer, "Efimov Physics in Cold Atoms", Ann. Phys. 322, 120 (2007).

[72] J. von Stecher, C.H. Greene, and D. Blume, "BEC-BCS crossover of a trapped two-component Fermi gas with unequal masses", Phys. Rev. A 76, 053613 (2007).

[73] D.R. Nelson and H.S. Seung, "Theory of melted flux liquids", Phys. Rev. B 48, 411 (1993). 
[74] W.R. Margo and D.M. Ceperley, "Ground state of two-dimensional Yukawa bosons: Applications to vortex melting", Phys. Rev. B 48, 411 (1993).

[75] H. Nordborg and G. Blatter, "Vortices and 2D Bosons: A Path-Integral Monte Carlo Study", Phys. Rev. Lett. 79, 1925 (1997).

[76] In the 2D regime achieved by confining the light-atom motion to zero point oscillations with amplitude $l_{0}$, the weakly bound molecular states exist at a negative $a$ satisafying the inequality $|a| \ll l_{0}$. See D.S. Petrov and G.V. Shlyapnikov, "Interatomic collisions in a tightly confined Bose gas", Phys. Rev. A 64, 012706 (2001).

[77] L. Xing, "Monte Carlo simulations of a two-dimensional hard-disk boson system", Phys. Rev. B 42, 8426 (1990).

[78] S. Wessel and M. Troyer, "Supersolid hard-core bosons on the triangular lattice", Phys. Rev. Lett. 95, 127205 (2005).

[79] D. Heidarian and K. Damle, "Persistent supersolid phase of hard-core bosons on the triangular lattice", Phys. Rev. Lett. 95, 127206 (2005).

[80] R.G. Melko, A. Paramekanti, A. A. Burkov, A. Vishwanath, D. N. Sheng, and L. Balents, "Supersolid order from disorder: hard-core bosons on the triangular lattice", Phys. Rev. Lett. 95, 127207 (2005).

[81] L. D. Carr, G. V. Shlyapnikov, and Y. Castin, "Achieving a BCS transition in an atomic Fermi gas", Phys. Rev. Lett. 92, 150404 (2004).

[82] C. Menotti, P. Pedri, and S. Stringari, "Expansion of an interacting Fermi gas", Phys. Rev. Lett. 89, 250402 (2002).

[83] K. M. O'Hara, S. L. Hemmer, M. E. Gehm, S. R. Granade, and J. E. Thomas, "Observation of a strongly interacting degenerate Fermi gas of atoms", Science 298, 2179 (2002).

[84] J. Kinast, S. L. Hemmer, M. E. Gehm, A. Turlapov, and J. E. Thomas, "Evidence for superfluidity in a resonantly interacting Fermi gas", Phys. Rev. Lett. 92, 150402 (2004).

[85] A. J. Kerman, J. M. Sage, S. Sainis, T. Bergeman, and D. DeMille, "Production and state-selective detection of ultracold RbCs molecules", Phys. Rev. Lett. 92, 153001 (2004).

[86] J. M. Sage, S. Sainis, T. Bergeman, and D. DeMille, "Optical production of ultracold polar molecules", Phys. Rev. Lett. 94, 203001 (2005).

[87] K. Winkler, F. Lang, G. Thalhammer, P. v. d. Straten, R. Grimm, and J.H. Denschlag, "Coherent optical transfer of Feshbach molecules to a lower vibrational state", Phys. Rev. Lett. 98, 043201 (2007). In this experiment the presence of an optical lattice suppressed inelastic collisions between 
molecules of bosonic ${ }^{87} \mathrm{Rb}$ atoms, which provided a highly efficient transfer of these molecules to a less excited ro-vibrational state and a long molecular lifetime of about 1 second.

[88] F. Lang, P. v.d. Straten, B. Brandstatter, G. Thalhammer, K. Winkler, P.S. Julienne, R. Grimm, and J.H. Denshlag, "Cruising through molecular bound-state manifolds with radiofrequency", Nature Physics 4, 223 (2008).

[89] L. Santos and T. Pfau, "Spin-3 chromium Bose-Einstein condensates", Phys. Rev. Lett. 96, 190404 (2006). 